J. Korean Math. Soc. 50 (2013), No. 5, pp. 1129-1163

http://dx.doi.org/10.4134/JKMS.2013.50.5.1129

\title{
ON THE LINEARIZATION OF DEFECT-CORRECTION METHOD FOR THE STEADY NAVIER-STOKES EQUATIONS
}

\author{
Yueqiang Shang, Do Wan Kim, and Tae-Chang Jo
}

\begin{abstract}
Based on finite element discretization, two linearization approaches to the defect-correction method for the steady incompressible Navier-Stokes equations are discussed and investigated. By applying $m$ times of Newton and Picard iterations to solve an artificial viscosity stabilized nonlinear Navier-Stokes problem, respectively, and then correcting the solution by solving a linear problem, two linearized defect-correction algorithms are proposed and analyzed. Error estimates with respect to the mesh size $h$, the kinematic viscosity $\nu$, the stability factor $\alpha$ and the number of nonlinear iterations $m$ for the discrete solution are derived for the linearized one-step defect-correction algorithms. Efficient stopping criteria for the nonlinear iterations are derived. The influence of the linearizations on the accuracy of the approximate solutions are also investigated. Finally, numerical experiments on a problem with known analytical solution, the lid-driven cavity flow, and the flow over a backwardfacing step are performed to verify the theoretical results and demonstrate the effectiveness of the proposed defect-correction algorithms.
\end{abstract}

\section{Introduction}

Let $\Omega$ be a bounded domain with Lipschitz-continuous boundary $\partial \Omega$ in $\mathbb{R}^{2}$. We consider the following steady incompressible Navier-Stokes problem:

$$
\begin{aligned}
-\nu \Delta u+(u \cdot \nabla) u+\nabla p=f & \text { in } \Omega, \\
\operatorname{div} u=0 & \text { in } \Omega,
\end{aligned}
$$

Received January 11, 2013; Revised June 11, 2013.

2010 Mathematics Subject Classification. 65N15, 65N30, 76D05, 76M10.

Key words and phrases. Navier-Stokes equations, finite element, defect-correction method, Newton iteration, Picard iteration.

This work was supported by the National Research Foundation (NRF) Grant funded by the Korean Government (MEST) (No. 2010-0017532). Y. Q. Shang was also supported by the Natural Science Foundation of China (No.11001061), the Project Sponsored by the Scientific Research Foundation for the Returned Overseas Chinese Scholars, State Education Ministry, and the Science and Technology Foundation of Guizhou Province of China (No. [2013]2212). D. W. Kim was supported by the National Research Foundation of Korea (NRF) funded by the Ministry of Education, Science and Technology (MEST) (No. 2011-0016748). T. C. Jo was supported by the Basic Science Research Program through the National Research Foundation of Korea funded by MEST (NRF-2010-0023265). 


$$
u=g \quad \text { on } \partial \Omega,
$$

where $u: \Omega \rightarrow \mathbb{R}^{2}$ is the velocity, $p: \Omega \rightarrow \mathbb{R}$ the pressure, $f: \Omega \rightarrow \mathbb{R}^{2}$ the prescribed body force, $g: \Omega \rightarrow \mathbb{R}^{2}$ the boundary velocity satisfying $\int_{\partial \Omega} g \cdot n d x=$ 0 (here $n$ denotes the unit outward normal vector to $\partial \Omega$ ), and $\nu$ the kinematic viscosity. Given a characteristic length $L$ and a characteristic velocity $U$, the Reynolds number is defined as $R e=U L / \nu$.

If $g \neq 0$ in equations (1.1)-(1.3), according to Temam [25] and He [11], we can introduce a vector function $\psi$ satisfying

$$
\operatorname{div} \psi=0 \text { in } \Omega, \psi=g \text { on } \partial \Omega,
$$

and

$$
\|(\psi \cdot \nabla) v\|_{X^{\prime}} \text { and }\|(v \cdot \nabla) \psi\|_{X^{\prime}} \text { are small, } \forall x \in X,
$$

where $X^{\prime}$ is the dual space of $X=H_{0}^{1}(\Omega)^{2}$. Then by setting $\widehat{u}=u-\psi,(1.1)$ (1.3) can be converted into a modified incompressible Navier-Stokes problem with new variables $(\widehat{u}, p)$ subject to homogeneous boundary conditions. Hence, for the sake of simplicity, we assume that $g=0$ in (1.1)-(1.3). For the functional setting of the Navier-Stokes equations, we refer the readers to Section 2.

Among numerical methods for the Navier-Stokes equations, the finite element methods have been a class of popular methods (cf. [3, 9, 10]). However, it is still challenging for the standard finite element method to simulate high Reynolds number flows. For high Reynolds number flows, due to the domination of the convection, spurious oscillations may occur in numerical simulations if the underlying mesh for the discretization of the equations is not fine enough. What is worse, the iterative method used to solve the nonlinear system may fail to converge (cf. $[4,19])$. To circumvent this difficulty, Layton et al. [18, 19] proposed a defect-correction method for the steady incompressible Navier-Stokes equations at high Reynolds numbers, in which an artificial viscosity term is added to the Navier-Stokes equations as a stability term. They first solve the artificial viscosity stabilized nonlinear Navier-Stokes equations on a relatively coarse mesh, and then correct the resultant residual by solving a linear problem on the same mesh. Numerical tests demonstrated the effectiveness of the defect-correction method. Subsequently, the method was combined with the adaptive technique [5], the subgrid stabilization method [16], and the twolevel method [21]. It was also applied to the viscoelastic fluid flow problems [20], the time-dependent Navier-Stokes equations [17], and the time-dependent conduction-convection problem [22, 23]. We refer to [16] and [22] for a literature review for the defect-correction method. However, on the issue that how to solve the artificial viscosity stabilized nonlinear Navier-Stokes problem, there are no any discussions in the above mentioned literature. All the numerical analyses and error estimates were based on the assumption that the artificial viscosity stabilized nonlinear problem is solved exactly (unfortunately, this is not the case in practical calculations). To the authors' knowledge, there is only one paper [26] that slightly involved this issue based on Oseen iterations for 
the nonlinear problem. However, it employed a partial linearization approach in the correction steps, which differs from our paper where a full linearization is used.

This paper concerns on the solving of the artificial viscosity stabilized nonlinear Navier-Stokes problem. Particularly, three issues are addressed: (i) what stability (and/or convergence) condition is needed when an iterative method such as Newton and Picard type iterations is applied to this continuous nonlinear system? (ii) what is the suitable and reliable stopping criterion when an iterative method is used to solve this nonlinear system? and (iii) what is the influence of the nonlinear iterations on the accuracy of the approximate solutions? By applying Newton and Picard iterations to the artificial viscosity stabilized nonlinear Navier-Stokes problem, respectively, we propose two linearized defect-correction algorithms based on finite element discretization. The key idea of the algorithms is to first solve an artificial viscosity stabilized nonlinear Navier-Stokes problem by applying $m$ times of Newton or Picard iteration, and then correct the resultant residual in several steps where only a linear problem is solved at each step.

The developed numerical algorithms can be casted into the framework of defect-correct method of Layton [19]. However, as mentioned above, our emphasis is put on the linearization approaches to the defect-correction method, the convergence conditions and the stopping criteria for the nonlinear iterations (or linearizations) and their influence on the accuracy of the approximate solutions, which were not discussed in [19]. We analyze the stability and convergence of the developed algorithms, and provide error estimates with respect to the mesh size $h$, the kinematic viscosity $\nu$, the stability factor $\alpha$ and the number of nonlinear iterations $m$ for the discrete velocity and pressure for onestep defect-correction algorithms. Moreover, it was commonly believed that $s$-step corrections in the defect-correction method are enough from the optimally asymptotical error point of view (see, e.g., $[16,17]$ ), where $s$ is the local polynomial degree of finite element subspace for the velocity. However, our present study shows that in small $\lambda$ cases (see (2.5) for the definition of $\lambda$ ), a little more than $s$ steps corrections are necessary for a good approximate solution (see Remark 4.5 and the numerical results in Section 5.3).

The paper is organized as follows. In the coming section, the functional setting of the Navier-Stokes problem and its finite element approximations are provided. In Section 3, based on finite element discretization, two linearization approaches to the defect-correction method for the Navier-Stokes equations are discussed and, subsequently, two linearized defect-correction algorithms are proposed. Stability analysis and error estimates are presented in Section 4. Section 5 is devoted to numerical experiments which verify the developed theory and demonstrate the effectiveness of the proposed algorithms. Finally, the article is concluded in Section 6 . 


\section{Preliminaries}

As usual, for a nonnegative integer $l$, we denote by $H^{l}(\Omega)$ the Sobolev space of functions with square integrable derivatives up to order $l$ in $\Omega$, equipped with the standard norm $\|\cdot\|_{l}$; while denote by $H_{0}^{1}(\Omega)$ the closed subspace of $H^{1}(\Omega)$ consisting of functions with zero trace on $\partial \Omega$; see, e.g., [1]. Throughout this paper, we shall use the letter $c$ or $C$ to denote a generic positive constant which is independent of the mesh parameter and may stand for different values at its different occurrences.

\subsection{Functional setting of the Navier-Stokes equations}

To introduce the variational formulation of problem (1.1)-(1.3) with $g=0$, we set

$$
X=H_{0}^{1}(\Omega)^{2}, Y=L^{2}(\Omega)^{2}, M=L_{0}^{2}(\Omega)=\left\{q \in L^{2}(\Omega): \int_{\Omega} q d x=0\right\},
$$

and define $a(\cdot, \cdot), d(\cdot, \cdot), b(\cdot, \cdot, \cdot)$ as

$$
\begin{aligned}
a(u, v) & =(\nabla u, \nabla v), d(v, q)=(\operatorname{div} v, q), \forall u, v \in X, q \in M, \\
b(u, v, w) & =((u \cdot \nabla) v, w)+\frac{1}{2}((\operatorname{div} u) v, w) \\
& =\frac{1}{2}((u \cdot \nabla) v, w)-\frac{1}{2}((u \cdot \nabla) w, v), \forall u, v, w \in X,
\end{aligned}
$$

where $(\cdot, \cdot)$ is the standard inner-product of $L^{2}(\Omega)$ or $L^{2}(\Omega)^{2}$.

It is well known that the trilinear term $b(\cdot, \cdot, \cdot)$ has the following properties (cf. $[9,15])$ :

$$
\begin{aligned}
& b(u, v, w)=-b(u, w, v), \forall u, v, w \in X \\
& |b(u, v, w)| \leq N\|\nabla u\|_{0}\|\nabla v\|_{0}\|\nabla w\|_{0}, \forall u, v, w \in X,
\end{aligned}
$$

where $N$ is a positive constant only depending on $\Omega$.

With the above notations, the variational formulation of (1.1)-(1.3) reads: find a pair $(u, p) \in X \times M$ such that

$$
\nu a(u, v)+b(u, u, v)-d(v, p)+d(u, q)=(f, v), \forall(v, q) \in X \times M .
$$

We make a regularity assumption on $\Omega$ and the following Stokes problem:

$$
-\nu \Delta v+\nabla q=f, \operatorname{div} v=0 \text { in } \Omega, v=0 \text { on } \partial \Omega .
$$

A0. Regularity. Given $f \in H^{k-1}(\Omega)^{2}(k=0,1,2)$, we assume that the solution pair $(v, q)$ of the Stokes problem (2.4) satisfies the following regularity result:

$$
\|v\|_{k+1}+\|q\|_{k} \leq c\|f\|_{k-1}, \quad k=0,1,2 .
$$

Under the above assumption, we have the following existence and uniqueness results (cf. $[9,13,25])$. 
Theorem 2.1. Given $f \in H^{k-1}(\Omega)^{2}$, if assumption $A 0$ and the following condition hold:

$$
\lambda=1-\frac{N\|f\|_{-1}}{\nu^{2}}>0,
$$

then problem (2.3) admits a unique solution pair $(u, p) \in\left(H^{k+1}(\Omega)^{2} \cap H_{0}^{1}(\Omega)^{2}\right) \times$ $\left(H^{k}(\Omega) \cap L_{0}^{2}(\Omega)\right)$ satisfying

$$
\begin{array}{r}
\|\nabla u\|_{0} \leq \nu^{-1}\|f\|_{-1},\|f\|_{-1}=\sup _{v \in X, v \neq 0} \frac{|(f, v)|}{\|\nabla v\|_{0}}, \\
\nu\|u\|_{k+1}+\|p\|_{k} \leq c\|f\|_{k-1}, \quad k=0,1,2 .
\end{array}
$$

\subsection{Finite element approximations}

Assume $T^{h}(\Omega)=\{K\}$ to be a uniformly regular triangulation (see, e.g., [9]) of $\Omega$ with mesh size $0<h<1, X_{h} \subset H_{0}^{1}(\Omega)^{2}, M_{h} \subset L_{0}^{2}(\Omega)$ two finite element spaces associated with $T^{h}(\Omega)$ and satisfying the following assumptions.

A1. Approximation. For each $(u, p) \in H^{k+1}(\Omega)^{2} \times H^{k}(\Omega)(k=0,1,2)$, there exists an approximation $\left(\pi_{h} u, \rho_{h} p\right) \in X_{h} \times M_{h}$ such that

$$
\left\|u-\pi_{h} u\right\|_{1} \leq c h^{s}\|u\|_{1+s}, \quad\left\|p-\rho_{h} p\right\|_{0} \leq c h^{s}\|p\|_{s}, \quad 0 \leq s \leq k .
$$

A2. Inf-sup condition. There exists a constant $\beta>0$ such that

$$
\beta\|q\|_{0} \leq \sup _{v \in X_{h}, v \neq 0} \frac{(\operatorname{div} v, q)}{\|\nabla v\|_{0}}, \forall q \in M_{h}
$$

We define

$V=\{v \in X: d(v, q)=0, \forall q \in M\}, V_{h}=\left\{v_{h} \in X_{h}: d\left(v_{h}, q\right)=0, \forall q \in M_{h}\right\}$.

It is well known that $[8,9]$

$$
\inf _{v_{h} \in V_{h}}\left\|\nabla\left(v-v_{h}\right)\right\|_{0} \leq C\left(1+\frac{1}{\beta}\right) \inf _{v_{h} \in X_{h}}\left\|\nabla\left(v-v_{h}\right)\right\|_{0}, \forall v \in V .
$$

Now, we give two examples of finite element subspaces satisfying assumptions $A 1$ and $A 2$. For a nonnegative integer $s$, we denote by $P_{s}$ the space of polynomials of degree less than or equal to $s$.

Example 2.1 (Mini elements [2]).

$$
\begin{aligned}
& X_{h}=\left\{v \in X:\left.v\right|_{K} \in\left(P_{1} \oplus \operatorname{span}\{b(K)\}\right)^{2}, \forall K \in T^{h}(\Omega)\right\}, \\
& M_{h}=\left\{q \in M \cap C^{0}(\Omega):\left.q\right|_{K} \in P_{1}, \forall K \in T^{h}(\Omega)\right\},
\end{aligned}
$$

where $b(K)$, called a cubic bubble function on the triangle $K$, is a cubic polynomial that vanishes on the edges of $K$.

Example 2.2 (Taylor-Hood elements [24]).

$$
\begin{aligned}
& X_{h}=\left\{v \in X:\left.v\right|_{K} \in\left(P_{2}\right)^{2}, \forall K \in T^{h}(\Omega)\right\}, \\
& M_{h}=\left\{q \in M \cap C^{0}(\Omega):\left.q\right|_{K} \in P_{1}, \forall K \in T^{h}(\Omega)\right\} .
\end{aligned}
$$


Under the above assumptions, the mixed finite element approximation of problem (2.3) reads: find a pair $\left(u_{h}, p_{h}\right) \in X_{h} \times M_{h}$ such that

(2.11) $\nu a\left(u_{h}, v\right)+b\left(u_{h}, u_{h}, v\right)-d\left(v, p_{h}\right)+d\left(u_{h}, q\right)=(f, v), \forall(v, q) \in X_{h} \times M_{h}$.

The following results on $\left(u_{h}, p_{h}\right)$ are classical (cf. $\left.[9,13,25]\right)$.

Theorem 2.2. Suppose that assumptions A1-A2 and the conditions of Theorem 2.1 hold. Then there exists a small $h_{0}>0$ such that for all $h \in\left(0, h_{0}\right]$, problem (2.11) admits a unique solution $\left(u_{h}, p_{h}\right)$ satisfying

$$
\begin{array}{r}
\left\|\nabla u_{h}\right\|_{0} \leq \nu^{-1}\|f\|_{-1}, \quad\left\|p_{h}\right\|_{0} \leq 3 \beta^{-1}\|f\|_{-1}, \\
\nu\left\|\nabla\left(u-u_{h}\right)\right\|_{0}+\left\|p-p_{h}\right\|_{0} \leq c h^{s}\|f\|_{s-1}, \quad 1 \leq s \leq k, \quad k=1,2 .
\end{array}
$$

Throughout this paper, we assume that Theorems 2.1 and 2.2 hold.

\section{Linearized defect-correction methods}

In this section, we shall first briefly describe the defect-correction method for the steady Navier-Stokes equations, and then propose two linearized defectcorrection algorithms by applying Newton and Picard iterations to the nonlinear system, respectively.

\subsection{The defect-correction method}

The defect-correction method is an iterative improvement technique which consists of an initial defect step followed by $s$-correction steps, where $s$ is the degree of local polynomials in finite element subspace for the velocity (see, e.g., [19]). In the defect step, the nonlinear Navier-Stokes equations with an added artificial viscosity term are solved. The solution is then corrected in the following steps, where only a linear problem is solved at each correction step. It incorporates the artificial viscosity term as a stabilizing factor, making the nonlinear system easier to resolve.

Defect step. Find $\left(u_{h}^{0}, p_{h}^{0}\right) \in X_{h} \times M_{h}$ such that

$$
\begin{aligned}
& (\alpha+\nu) a\left(u_{h}^{0}, v\right)+b\left(u_{h}^{0}, u_{h}^{0}, v\right)-d\left(v, p_{h}^{0}\right)+d\left(u_{h}^{0}, q\right) \\
= & (f, v), \forall(v, q) \in X_{h} \times M_{h},
\end{aligned}
$$

where $\alpha<1$ is a user-selected stability parameter and typically, chosen as $\alpha=\mathcal{O}(h)$.

The resulting defect or residual $\mathcal{R}\left(u_{h}^{0}, p_{h}^{0}\right)$ for problem (3.1) is defined as

$$
\left(\mathcal{R}\left(u_{h}^{0}, p_{h}^{0}\right), v\right)=(f, v)-\nu a\left(u_{h}^{0}, v\right)-b\left(u_{h}^{0}, u_{h}^{0}, v\right)+d\left(v, p_{h}^{0}\right)-d\left(u_{h}^{0}, q\right) .
$$

We compute the correction $\left(\epsilon_{h}^{0}, \eta_{h}^{0}\right)$ by solving the following linear problem

$$
\begin{aligned}
& (\alpha+\nu) a\left(\epsilon_{h}^{0}, v\right)+b\left(\epsilon_{h}^{0}, u_{h}^{0}, v\right)+b\left(u_{h}^{0}, \epsilon_{h}^{0}, v\right)-d\left(v, \eta_{h}^{0}\right)+d\left(\epsilon_{h}^{0}, q\right) \\
= & \left(\mathcal{R}\left(u_{h}^{0}, p_{h}^{0}\right), v\right), \quad \forall(v, q) \in X_{h} \times M_{h},
\end{aligned}
$$


and then update the solution $\left(u_{h}^{1}, p_{h}^{1}\right)=\left(u_{h}^{0}+\epsilon_{h}^{0}, p_{h}^{0}+\eta_{h}^{0}\right)$. It is expected that $\left(u_{h}^{1}, p_{h}^{1}\right)$ becomes a better approximation to $\left(u_{h}, p_{h}\right)$ than $\left(u_{h}^{0}, p_{h}^{0}\right)$. Combining (3.2) with (3.3), we get that the solution $\left(u_{h}^{1}, p_{h}^{1}\right)$ satisfies

$$
\begin{aligned}
& (\alpha+\nu) a\left(u_{h}^{1}, v\right)+b\left(u_{h}^{1}, u_{h}^{0}, v\right)+b\left(u_{h}^{0}, u_{h}^{1}, v\right)-d\left(v, p_{h}^{1}\right)+d\left(u_{h}^{1}, q\right) \\
= & (f, v)+b\left(u_{h}^{0}, u_{h}^{0}, v\right)+\alpha a\left(u_{h}^{0}, v\right), \quad \forall(v, q) \in X_{h} \times M_{h} .
\end{aligned}
$$

In general, for each $j(1 \leq j \leq s)$, the defect $\mathcal{R}\left(u_{h}^{j}, p_{h}^{j}\right)$ and correction $\left(\epsilon_{h}^{j}, \eta_{h}^{j}\right)$ are defined, respectively, by

$$
\left(\mathcal{R}\left(u_{h}^{j}, p_{h}^{j}\right), v\right)=(f, v)-\nu a\left(u_{h}^{j}, v\right)-b\left(u_{h}^{j}, u_{h}^{j}, v\right)+d\left(v, p_{h}^{j}\right)-d\left(u_{h}^{j}, q\right),
$$

and

$$
\begin{aligned}
& (\alpha+\nu) a\left(\epsilon_{h}^{j}, v\right)+b\left(\epsilon_{h}^{j}, u_{h}^{j}, v\right)+b\left(u_{h}^{j}, \epsilon_{h}^{j}, v\right)-d\left(v, \eta_{h}^{j}\right)+d\left(\epsilon_{h}^{j}, q\right) \\
= & \left(\mathcal{R}\left(u_{h}^{j}, p_{h}^{j}\right), v\right), \quad \forall(v, q) \in X_{h} \times M_{h},
\end{aligned}
$$

and the solution $\left(u_{h}^{j+1}, p_{h}^{j+1}\right)$ is defined as $\left(u_{h}^{j+1}, p_{h}^{j+1}\right)=\left(u_{h}^{j}+\epsilon_{h}^{j}, p_{h}^{j}+\eta_{h}^{j}\right)$. It is noted that the solution $\left(u_{h}^{j+1}, p_{h}^{j+1}\right)$ satisfies from (3.5) and (3.6) that

$$
\begin{aligned}
& (\alpha+\nu) a\left(u_{h}^{j+1}, v\right)+b\left(u_{h}^{j+1}, u_{h}^{j}, v\right)+b\left(u_{h}^{j}, u_{h}^{j+1}, v\right)-d\left(v, p_{h}^{j+1}\right)+d\left(u_{h}^{j+1}, q\right) \\
= & (f, v)+b\left(u_{h}^{j}, u_{h}^{j}, v\right)+\alpha a\left(u_{h}^{j}, v\right), \quad \forall(v, q) \in X_{h} \times M_{h} .
\end{aligned}
$$

Therefore, we have the following s-correction steps.

$s$-correction steps. For $j=1,2, \ldots, s$, find $\left(u_{h}^{j}, p_{h}^{j}\right) \in X_{h} \times M_{h}$ such that

$$
\begin{aligned}
& (\alpha+\nu) a\left(u_{h}^{j}, v\right)+b\left(u_{h}^{j}, u_{h}^{j-1}, v\right)+b\left(u_{h}^{j-1}, u_{h}^{j}, v\right)-d\left(v, p_{h}^{j}\right)+d\left(u_{h}^{j}, q\right) \\
= & (f, v)+b\left(u_{h}^{j-1}, u_{h}^{j-1}, v\right)+\alpha a\left(u_{h}^{j-1}, v\right), \quad \forall(v, q) \in X_{h} \times M_{h} .
\end{aligned}
$$

For the defect step, by a standard argument (cf. $[9,16,19])$, we have the following results.

Theorem 3.1. Assume that $(u, p)$ is the solution of problem $(2.3)$ and $\left(u_{h}^{0}, p_{h}^{0}\right)$ is the solution of problem (3.1), then the following estimates hold

$$
\begin{aligned}
&\left\|\nabla u_{h}^{0}\right\|_{0} \leq(\alpha+\nu)^{-1}\|f\|_{-1}, \quad\left\|p_{h}^{0}\right\|_{0} \leq 3 \beta^{-1}\|f\|_{-1}, \\
& \nu\left\|\nabla\left(u-u_{h}^{0}\right)\right\|_{0} \leq C(\beta)\left(1+3 \lambda^{-1}\right) \nu \inf _{w_{h} \in X_{h}}\left\|\nabla\left(u-w_{h}\right)\right\|_{0} \\
&+\sqrt{2} \lambda^{-1} \inf _{q_{h} \in M_{h}}\left\|p-q_{h}\right\|_{0}+\lambda^{-1} \frac{\alpha}{\alpha+\nu}\|f\|_{-1},
\end{aligned}
$$

$$
\begin{aligned}
\left\|p-p_{h}^{0}\right\|_{0} \leq & C(\beta)\left(1+3 \lambda^{-1}\right) \nu \inf _{w_{h} \in X_{h}}\left\|\nabla\left(u-w_{h}\right)\right\|_{0} \\
& +\left(1+\frac{3 \sqrt{2}}{\lambda \beta}\right) \inf _{q_{h} \in M_{h}}\left\|p-q_{h}\right\|_{0}+\frac{1+3 \lambda^{-1}}{\beta} \frac{\alpha}{\alpha+\nu}\|f\|_{-1} .
\end{aligned}
$$


Proof. The first result of (3.8) is obtained by taking $v=u_{h}^{0}$ in (3.1) and applying (2.1). The second result of (3.8) follows from (3.1) with $q=0$, the inf-sup condition and the uniqueness condition (2.5). While (3.9) and (3.10) can be obtained by a standard argument together with the uniqueness condition (2.5) and (2.10); see, e.g., [16, 19].

\subsection{Linearized defect-correction algorithms}

By applying $m$ times of Newton and Picard iterations to the nonlinear problem (3.1), respectively, we develop the following two linearized defect-correction algorithms.

Algorithm 1. Newton iterative defect-correction algorithm.

Step 1. Find a stabilized finite element iterative solution $\left(u_{h 0}^{m}, p_{h 0}^{m}\right) \in X_{h} \times$ $M_{h}$ such that

$$
\begin{aligned}
& (\alpha+\nu) a\left(u_{h 0}^{n}, v\right)+b\left(u_{h 0}^{n}, u_{h 0}^{n-1}, v\right)+b\left(u_{h 0}^{n-1}, u_{h 0}^{n}, v\right) \\
& -d\left(v, p_{h 0}^{n}\right)+d\left(u_{h 0}^{n}, q\right) \\
= & (f, v)+b\left(u_{h 0}^{n-1}, u_{h 0}^{n-1}, v\right), \forall(v, q) \in X_{h} \times M_{h},
\end{aligned}
$$

for $n=1,2, \ldots, m$.

Step 2. For $j=1,2, \ldots, s$, find $\left(u_{m h}^{j}, p_{m h}^{j}\right) \in X_{h} \times M_{h}$ such that

$$
\begin{aligned}
& (\alpha+\nu) a\left(u_{m h}^{j}, v\right)+b\left(u_{m h}^{j}, u_{m h}^{j-1}, v\right)+b\left(u_{m h}^{j-1}, u_{m h}^{j}, v\right) \\
& -d\left(v, p_{m h}^{j}\right)+d\left(u_{m h}^{j}, q\right) \\
= & (f, v)+b\left(u_{m h}^{j-1}, u_{m h}^{j-1}, v\right)+\alpha a\left(u_{m h}^{j-1}, v\right), \forall(v, q) \in X_{h} \times M_{h},
\end{aligned}
$$

with $\left(u_{m h}^{0}, p_{m h}^{0}\right)=\left(u_{h 0}^{m}, p_{h 0}^{m}\right)$.

Algorithm 2. Picard iterative defect-correction algorithm.

Step 1. Find a stabilized finite element solution $\left(u_{h 0}^{m}, p_{h 0}^{m}\right) \in X_{h} \times M_{h}$ by the following iterative procedure:

$$
\begin{aligned}
& (\alpha+\nu) a\left(u_{h 0}^{n}, v\right)+b\left(u_{h 0}^{n-1}, u_{h 0}^{n}, v\right)-d\left(v, p_{h 0}^{n}\right)+d\left(u_{h 0}^{n}, q\right) \\
= & (f, v), \quad \forall(v, q) \in X_{h} \times M_{h}
\end{aligned}
$$

for $n=1,2, \ldots, m$.

Step 2. The same as Algorithm 1.

In the above algorithms, the initial guess $u_{h 0}^{0}$ for the nonlinear iterations in the defect step can be defined by the following Stokes equations

$$
(\alpha+\nu) a\left(u_{h 0}^{0}, v\right)-d\left(v, p_{h 0}^{0}\right)+d\left(u_{h 0}^{0}, q\right)=(f, v), \forall(v, q) \in X_{h} \times M_{h},
$$

the parameter $m$ is the nonlinear iterations count satisfying a certain stopping criterion. 


\section{Error estimates}

Note that the defect step of Algorithms 1 and 2 are just the Newton and Picard iterations applied to the artificial viscosity stabilized nonlinear NavierStokes equations (3.1). Following the framework of [12] where classical iterative methods for the 2D incompressible Navier-Stokes equations were analyzed, we can get the following Lemmas 4.1-4.2.

Lemma 4.1. Under the following condition

$$
\frac{N}{(\alpha+\nu)^{2}}\|f\|_{-1}<\frac{3}{25},
$$

the iterative scheme (3.11) is stable and convergent. Moreover, the iterative solution $\left(u_{h 0}^{m}, p_{h 0}^{m}\right)$ satisfies

$$
\begin{aligned}
& (\alpha+\nu)\left\|\nabla u_{h 0}^{m}\right\|_{0} \leq \frac{5}{3}\|f\|_{-1}, \quad\left\|p_{h 0}^{m}\right\|_{0} \leq \frac{11}{3 \beta}\|f\|_{-1}, m \geq 0 \\
& (\alpha+\nu)\left\|\nabla\left(u_{h}^{0}-u_{h 0}^{m}\right)\right\|_{0} \leq\left(\frac{5 N}{4(\alpha+\nu)^{2}}\|f\|_{-1}\right)^{2^{m}-1}\|f\|_{-1}, m \geq 0 \\
& \left\|p_{h}^{0}-p_{h 0}^{m}\right\|_{0} \leq 3 \beta^{-1}\left(\frac{5 N}{4(\alpha+\nu)^{2}}\|f\|_{-1}\right)^{2^{m}-1}\|f\|_{-1}, m \geq 0 \\
& (\alpha+\nu)\left\|\nabla\left(u_{h}^{0}-u_{h 0}^{m}\right)\right\|_{0}+\beta\left\|p_{h}^{0}-p_{h 0}^{m}\right\|_{0} \\
& \leq c|\log h|^{\frac{1}{2}}\left\|\nabla\left(u_{h 0}^{m}-u_{h 0}^{m-1}\right)\right\|_{0}\left\|u_{h 0}^{m}-u_{h 0}^{m-1}\right\|_{0}, m \geq 1 \\
& \lim _{m \rightarrow \infty}\left(\left\|\nabla\left(u_{h 0}^{m}-u_{h 0}^{m-1}\right)\right\|_{0}+\left\|u_{h 0}^{m}-u_{h 0}^{m-1}\right\|_{0}\right)=0 .
\end{aligned}
$$

Lemma 4.2. Assume that the following condition hold:

$$
\frac{N}{(\alpha+\nu)^{2}}\|f\|_{-1}<1 .
$$

Then the iterative scheme (3.13) is stable and convergent. Moreover, the iterative solution $\left(u_{h 0}^{m}, p_{h 0}^{m}\right)$ satisfies

$$
\begin{aligned}
& (\alpha+\nu)\left\|\nabla u_{h 0}^{m}\right\|_{0} \leq\|f\|_{-1}, \quad\left\|p_{h 0}^{m}\right\|_{0} \leq 3 \beta^{-1}\|f\|_{-1}, m \geq 0, \\
& (\alpha+\nu)\left\|\nabla\left(u_{h}^{0}-u_{h 0}^{m}\right)\right\|_{0} \leq\left(\frac{N}{(\alpha+\nu)^{2}}\|f\|_{-1}\right)^{m}\|f\|_{-1}, m \geq 0, \\
& \left\|p_{h}^{0}-p_{h 0}^{m}\right\|_{0} \leq 3 \beta^{-1}\left(\frac{N}{(\alpha+\nu)^{2}}\|f\|_{-1}\right)^{m}\|f\|_{-1}, m \geq 0, \\
& (\alpha+\nu)\left\|\nabla\left(u_{h}^{0}-u_{h 0}^{m}\right)\right\|_{0}+\beta\left\|p_{h}^{0}-p_{h 0}^{m}\right\|_{0} \\
& \leq c(\alpha+\nu)\left\|u_{h 0}^{m}-u_{h 0}^{m-1}\right\|_{0}, m \geq 1, \\
& \quad \lim _{m \rightarrow \infty}\left\|u_{h 0}^{m}-u_{h 0}^{m-1}\right\|_{0}=0 .
\end{aligned}
$$


Remark 4.1. From Lemmas 4.1-4.2, we can see that Algorithm 1 is of secondorder convergence rate with respect to the iterative step $m$. However, its stability condition is stricter than that of Algorithm 2. On the contrary, the stability condition of Algorithm 2 is weaker than the uniqueness condition (2.5), making it more applicable in the simulation of high Reynolds number flows.

Setting $\left(e^{j}, \eta^{j}\right)=\left(u-u_{m h}^{j}, p-p_{m h}^{j}\right)(j \geq 1)$ and similarly $\left(e^{0}, \eta^{0}\right)=(u-$ $\left.u_{m h}^{0}, p-p_{m h}^{0}\right)=\left(u-u_{h 0}^{m}, p-p_{h 0}^{m}\right)$, now we derive error estimates for the discrete velocity and pressure computed by the one-step defect-correction algorithms.

Theorem 4.1. Assume that $(u, p)$ is the solution to the Navier-Stokes equations (2.3). Under the conditions of Theorems 2.1, 2.2 and Lemma 4.1, the one-step correction solution $\left(u_{m h}^{1}, p_{m h}^{1}\right)$ obtained from Algorithm 1 satisfies the following error estimates:

$$
\begin{aligned}
& \nu\left\|\nabla\left(u-u_{m h}^{1}\right)\right\|_{0} \\
\leq & c(\beta)\left(1+\frac{1}{\lambda}\right)\|f\|_{s-1} h^{s}+c(\beta)\left(1+\frac{1}{\lambda}+\frac{1}{\lambda^{2}}\right) \frac{\|f\|_{s-1}^{2}}{\|f\|_{-1}} h^{2 s} \\
& +\frac{5 \alpha}{16 \lambda \nu}\|f\|_{-1}+\frac{3 \alpha^{2}}{5 \lambda^{2} \nu(\alpha+\nu)}\|f\|_{-1} \\
+ & \frac{5}{16}\left(\frac{5 N}{4(\alpha+\nu)^{2}}\|f\|_{-1}\right)^{2^{m}-1}\|f\|_{-1} \\
+ & \frac{3}{5}\left(\frac{5 N}{4(\alpha+\nu)^{2}}\|f\|_{-1}\right)^{2^{m+1}-2}\|f\|_{-1}, m \geq 0,1 \leq s \leq k, k=1,2
\end{aligned}
$$

(4.14) $\left\|p-p_{m h}^{1}\right\|_{0}$

$$
\begin{aligned}
\leq & c(\beta)\left(1+\frac{\alpha}{\nu}+\frac{\alpha}{\lambda \nu}\right)\|f\|_{s-1} h^{s}+c(\beta)\left(1+\frac{1}{\lambda}+\frac{1}{\lambda^{2}}\right) \frac{\|f\|_{s-1}^{2}}{\|f\|_{-1}} h^{2 s} \\
& +\frac{11 \alpha^{2}}{4 \lambda \nu(\alpha+\nu) \beta}\|f\|_{-1}+\frac{33 \alpha^{2}}{25 \lambda^{2} \nu^{2} \beta}\|f\|_{-1} \\
& +\frac{11}{4 \beta}\left(\frac{5 N}{4(\alpha+\nu)^{2}}\|f\|_{-1}\right)^{2^{m}-1}\|f\|_{-1} \\
& +\frac{33}{25 \beta}\left(\frac{5 N}{4(\alpha+\nu)^{2}}\|f\|_{-1}\right)^{2^{m+1}-2}\|f\|_{-1}, m \geq 0,1 \leq s \leq k, k=1,2 .
\end{aligned}
$$

Before giving the proof of Theorem 4.1, we make some remarks.

Remark 4.2. The estimators (4.13) and (4.14) consist of six terms. The first two terms relate to the usual finite element discretization error. The second two terms are the defect-correction method error introduced by the artificial viscosity stability term. While the last two terms are the nonlinear iteration (or linearization) error in solving the nonlinear system, which is an interesting result indicating the influence of the nonlinear iterations on the accuracy of 
the approximate solutions. It shows that the nonlinear solver should converge sufficiently for a good approximate solution.

Remark 4.3. The error factor $\frac{5 N}{4(\alpha+\nu)^{2}}\|f\|_{-1}$ in the estimators shows that for a given problem (i.e., when the Reynolds number is fixed), the bigger the artificial viscosity stability factor $\alpha$ is, the faster the nonlinear iterations converge. However, as the second two terms in the estimators shown, when $\alpha$ grows, the defect-correction error increases. Therefore, to ensure the effectiveness of the algorithm, the parameter $\alpha$ should be carefully chosen.

Proof of Theorem 4.1. Subtracting (3.12) with $j=1$ from (2.3), we have

$$
\begin{aligned}
& (\alpha+\nu) a\left(e^{1}, v\right)+b(u, u, v)-b\left(u_{m h}^{1}, u_{m h}^{0}, v\right)-b\left(u_{m h}^{0}, u_{m h}^{1}, v\right) \\
& +b\left(u_{m h}^{0}, u_{m h}^{0}, v\right)-d\left(v, \eta^{1}\right)+d\left(e^{1}, q\right) \\
= & \alpha a\left(e^{0}, v\right), \forall(v, q) \in X_{h} \times M_{h} .
\end{aligned}
$$

Due to the fact that

$$
\begin{aligned}
& b(u, u, v)-b\left(u_{m h}^{1}, u_{m h}^{0}, v\right)-b\left(u_{m h}^{0}, u_{m h}^{1}, v\right)+b\left(u_{m h}^{0}, u_{m h}^{0}, v\right) \\
= & b(u, u, v)+b\left(e^{1}, u_{m h}^{0}, v\right)-b\left(u, u_{m h}^{0}, v\right)+b\left(u_{m h}^{0}, e^{1}, v\right) \\
& -b\left(u_{m h}^{0}, u, v\right)+b\left(u_{m h}^{0}, u_{m h}^{0}, v\right) \\
= & b\left(u, e^{0}, v\right)+b\left(e^{1}, u_{m h}^{0}, v\right)+b\left(u_{m h}^{0}, e^{1}, v\right)-b\left(u_{m h}^{0}, e^{0}, v\right) \\
= & b\left(e^{0}, e^{0}, v\right)+b\left(e^{1}, u_{m h}^{0}, v\right)+b\left(u_{m h}^{0}, e^{1}, v\right)
\end{aligned}
$$

we get

$$
\begin{aligned}
& (\alpha+\nu) a\left(e^{1}, v\right)+b\left(e^{1}, u_{m h}^{0}, v\right)+b\left(u_{m h}^{0}, e^{1}, v\right)-d\left(v, \eta^{1}\right)+d\left(e^{1}, q\right) \\
= & -b\left(e^{0}, e^{0}, v\right)+\alpha a\left(e^{0}, v\right), \forall(v, q) \in X_{h} \times M_{h} .
\end{aligned}
$$

It is noted that (4.16) is also valid for all $(v, q) \in V_{h} \times M_{h}$ since $V_{h} \subset X_{h}$. Let $\left(w_{h}, q_{h}\right)$ be an arbitrary functions pair in $V_{h} \times M_{h}$. By adding and subtracting appropriate terms in (4.16), we obtain

$$
\begin{aligned}
& (\alpha+\nu) a\left(w_{h}-u_{m h}^{1}, v\right)+b\left(w_{h}-u_{m h}^{1}, u_{m h}^{0}, v\right)+b\left(u_{m h}^{0}, w_{h}-u_{m h}^{1}, v\right) \\
= & -(\alpha+\nu) a\left(u-w_{h}, v\right)-b\left(u-w_{h}, u_{m h}^{0}, v\right) \\
& -b\left(u_{m h}^{0}, u-w_{h}, v\right)+d\left(v, q_{h}-p_{m h}^{1}\right) \\
& +d\left(v, p-q_{h}\right)-d\left(e^{1}, q\right)-b\left(e^{0}, e^{0}, v\right)+\alpha a\left(e^{0}, v\right), \forall(v, q) \in V_{h} \times M_{h} .
\end{aligned}
$$

Taking $(v, q)=\left(w_{h}-u_{m h}^{1}, 0\right)$ in the above equality and applying (2.1), (2.2), we arrive at

$$
\begin{aligned}
& \left(\alpha+\nu-N\left\|\nabla u_{m h}^{0}\right\|_{0}\right)\left\|\nabla\left(w_{h}-u_{m h}^{1}\right)\right\|_{0} \\
\leq & \left(\alpha+\nu+2 N\left\|\nabla u_{m h}^{0}\right\|_{0}\right)\left\|\nabla\left(u-w_{h}\right)\right\|_{0}+\sqrt{2}\left\|p-q_{h}\right\|_{0} \\
& +N\left\|\nabla e^{0}\right\|_{0}^{2}+\alpha\left\|\nabla e^{0}\right\|_{0} .
\end{aligned}
$$


From Theorem 3.1 and Lemma 4.1, we have

$$
\begin{gathered}
\text { (4.18) } \alpha+\nu-N\left\|\nabla u_{m h}^{0}\right\|_{0} \geq(\alpha+\nu)\left(1-\frac{5 N}{3(\alpha+\nu)^{2}}\|f\|_{-1}\right)>\frac{4}{5}(\alpha+\nu), \\
(4.19) \quad \alpha+\nu+2 N\left\|\nabla u_{m h}^{0}\right\|_{0} \leq(\alpha+\nu)\left(1+\frac{10 N}{3(\alpha+\nu)^{2}}\|f\|_{-1}\right)<\frac{7}{5}(\alpha+\nu), \\
(4.20) \quad \alpha\left\|\nabla e^{0}\right\|_{0} \leq C(\beta)\left(1+3 \lambda^{-1}\right) \alpha \inf _{w_{h} \in X_{h}}\left\|\nabla\left(u-w_{h}\right)\right\|_{0} \\
+\frac{\alpha \sqrt{2}}{\lambda \nu} \inf _{q_{h} \in M_{h}}\left\|p-q_{h}\right\|_{0}+\frac{\alpha^{2}}{\lambda \nu(\alpha+\nu)}\|f\|_{-1} \\
+\frac{\alpha}{\alpha+\nu}\left(\frac{5 N}{4(\alpha+\nu)^{2}}\|f\|_{-1}\right)^{2^{m}-1}\|f\|_{-1}, m \geq 0,
\end{gathered}
$$

(4.21) $\quad N\left\|\nabla e^{0}\right\|_{0}^{2} \leq C^{2}(\beta) 4 N\left(1+3 \lambda^{-1}\right)^{2}\left(\inf _{w_{h} \in X_{h}}\left\|\nabla\left(u-w_{h}\right)\right\|_{0}\right)^{2}$

$$
\begin{aligned}
& +\frac{8 N}{\lambda^{2} \nu^{2}}\left(\inf _{q_{h} \in M_{h}}\left\|p-q_{h}\right\|_{0}\right)^{2}+\frac{4 N \alpha^{2}}{\lambda^{2} \nu^{2}(\alpha+\nu)^{2}}\|f\|_{-1}^{2} \\
& +\frac{4 N}{(\alpha+\nu)^{2}}\left(\frac{5 N}{4(\alpha+\nu)^{2}}\|f\|_{-1}\right)^{2^{m+1}-2}\|f\|_{-1}^{2} \\
& \leq C^{2}(\beta) 4 N\left(1+3 \lambda^{-1}\right)^{2}\left(\inf _{w_{h} \in X_{h}}\left\|\nabla\left(u-w_{h}\right)\right\|_{0}\right)^{2} \\
& +\frac{8 N}{\lambda^{2} \nu^{2}}\left(\inf _{q_{h} \in M_{h}}\left\|p-q_{h}\right\|_{0}\right)^{2}+\frac{12 \alpha^{2}}{25 \lambda^{2} \nu^{2}}\|f\|_{-1} \\
& +\frac{12}{25}\left(\frac{5 N}{4(\alpha+\nu)^{2}}\|f\|_{-1}\right)^{2^{m+1}-2}\|f\|_{-1}, m \geq 0,
\end{aligned}
$$

where the stability condition (4.1) is used.

Combining (4.17) with (4.18)-(4.21) and the triangle inequality, taking the infimum over $w_{h} \in V_{h}, q_{h} \in M_{h}$, and using (2.10), we get

$$
\begin{aligned}
\nu\left\|\nabla\left(u-u_{m h}^{1}\right)\right\|_{0} \leq & C(\beta)\left(\frac{5\left(1+3 \lambda^{-1}\right) \alpha}{4(\alpha+\nu)}+\frac{11}{4}\right) \nu \inf _{w_{h} \in X_{h}}\left\|\nabla\left(u-w_{h}\right)\right\|_{0} \\
& +\frac{5 \sqrt{2}\left(\lambda^{-1} \alpha+\nu\right)}{4(\alpha+\nu)} \inf _{q_{h} \in M_{h}}\left\|p-q_{h}\right\|_{0} \\
& +C^{2}(\beta) \frac{5 N\left(1+3 \lambda^{-1}\right)^{2} \nu}{\alpha+\nu}\left(\inf _{w_{h} \in X_{h}}\left\|\nabla\left(u-w_{h}\right)\right\|_{0}\right)^{2} \\
& +\frac{10 N}{\lambda^{2} \nu(\alpha+\nu)}\left(\inf _{q_{h} \in M_{h}}\left\|p-q_{h}\right\|_{0}\right)^{2} \\
& +\frac{5 \alpha^{2}}{4 \lambda(\alpha+\nu)^{2}}\|f\|_{-1}+\frac{3 \alpha^{2}}{5 \lambda^{2} \nu(\alpha+\nu)}\|f\|_{-1}
\end{aligned}
$$




$$
\begin{aligned}
& +\frac{5 \nu \alpha}{4(\alpha+\nu)^{2}}\left(\frac{5 N}{4(\alpha+\nu)^{2}}\|f\|_{-1}\right)^{2^{m}-1}\|f\|_{-1} \\
& +\frac{3 \nu}{5(\alpha+\nu)}\left(\frac{5 N}{4(\alpha+\nu)^{2}}\|f\|_{-1}\right)^{2^{m+1}-2}\|f\|_{-1}, m \geq 0 .
\end{aligned}
$$

Therefore, the applications of Theorem 2.2 and the uniqueness condition (2.5) yield

$$
\begin{aligned}
\nu\left\|\nabla\left(u-u_{m h}^{1}\right)\right\|_{0} \leq & c(\beta)\left(1+\frac{1}{\lambda}\right)\|f\|_{s-1} h^{s}+c(\beta)\left(1+\frac{1}{\lambda}+\frac{1}{\lambda^{2}}\right) \frac{\|f\|_{s-1}^{2}}{\|f\|_{-1}} h^{2 s} \\
& +\frac{5 \alpha^{2}}{4 \lambda(\alpha+\nu)^{2}}\|f\|_{-1}+\frac{3 \alpha^{2}}{5 \lambda^{2} \nu(\alpha+\nu)}\|f\|_{-1} \\
& +\frac{5 \nu \alpha}{4(\alpha+\nu)^{2}}\left(\frac{5 N}{4(\alpha+\nu)^{2}}\|f\|_{-1}\right)^{2^{m}-1}\|f\|_{-1} \\
& +\frac{3 \nu}{5(\alpha+\nu)}\left(\frac{5 N}{4(\alpha+\nu)^{2}}\|f\|_{-1}\right)^{2^{m+1}-2}\|f\|_{-1}, \\
& m \geq 0,1 \leq s \leq k, k=1,2,
\end{aligned}
$$

which, together with the inequalities $(\alpha+\nu)^{2} \geq 4 \alpha \nu$ and $\frac{\nu}{\alpha+\nu}<1$, implies (4.13).

On the other hand, arbitrarily choosing $q_{h} \in M_{h}$, adding and subtracting appropriate terms in (4.16), we have

$$
\begin{aligned}
& d\left(v, q_{h}-p_{m h}^{1}\right) \\
= & (\alpha+\nu) a\left(e^{1}, v\right)+b\left(e^{1}, u_{m h}^{0}, v\right)+b\left(u_{m h}^{0}, e^{1}, v\right)-d\left(v, p-q_{h}\right) \\
& +d\left(e^{1}, q\right)+b\left(e^{0}, e^{0}, v\right)-\alpha a\left(e^{0}, v\right), \quad \forall(v, q) \in X_{h} \times M_{h} .
\end{aligned}
$$

Taking $q=0$ in the above equations, we deduce from the inf-sup condition (2.9), (2.2), (4.1)-(4.2) and (4.19)-(4.21) that

$$
\begin{aligned}
& \beta\left\|q_{h}-p_{m h}^{1}\right\|_{0} \\
\leq & (\alpha+\nu)\left\|\nabla e^{1}\right\|_{0}+2 N\left\|\nabla e^{1}\right\|_{0}\left\|\nabla u_{m h}^{0}\right\|_{0}+\sqrt{2}\left\|p-q_{h}\right\|_{0} \\
& +N\left\|\nabla e^{0}\right\|_{0}^{2}+\alpha\left\|\nabla e^{0}\right\|_{0} \\
\leq & \frac{7}{5}(\alpha+\nu)\left\|\nabla e^{1}\right\|_{0}+\sqrt{2}\left\|p-q_{h}\right\|_{0}+N\left\|\nabla e^{0}\right\|_{0}^{2}+\alpha\left\|\nabla e^{0}\right\|_{0} \\
\leq & C(\beta)\left(\frac{11}{4}\left(1+3 \lambda^{-1}\right) \alpha+\frac{77(\alpha+\nu)}{20}\right) \inf _{w_{h} \in X_{h}}\left\|\nabla\left(u-w_{h}\right)\right\|_{0} \\
& +\left(\frac{11 \sqrt{2} \alpha}{4 \lambda \nu}+\frac{11}{4} \sqrt{2}\right) \inf _{q_{h} \in M_{h}}\left\|p-q_{h}\right\|_{0} \\
& +C^{2}(\beta) 11 N\left(1+3 \lambda^{-1}\right)^{2}\left(\inf _{w_{h} \in X_{h}}\left\|\nabla\left(u-w_{h}\right)\right\|_{0}\right)^{2}
\end{aligned}
$$




$$
\begin{aligned}
& +\frac{22 N}{\lambda^{2} \nu^{2}}\left(\inf _{q_{h} \in M_{h}}\left\|p-q_{h}\right\|_{0}\right)^{2}+\frac{11 \alpha^{2}}{4 \lambda \nu(\alpha+\nu)}\|f\|_{-1} \\
& +\frac{33 \alpha^{2}}{25 \lambda^{2} \nu^{2}}\|f\|_{-1}+\frac{11 \alpha}{4(\alpha+\nu)}\left(\frac{5 N}{4(\alpha+\nu)^{2}}\|f\|_{-1}\right)^{2^{m}-1}\|f\|_{-1} \\
& +\frac{33}{25}\left(\frac{5 N}{4(\alpha+\nu)^{2}}\|f\|_{-1}\right)^{2^{m+1}-2}\|f\|_{-1}, m \geq 0 .
\end{aligned}
$$

Thus, applying the triangle inequality, Theorem 2.2 and the uniqueness condition (2.5), we obtain

$$
\begin{aligned}
& \left\|p-p_{m h}^{1}\right\|_{0} \\
\leq & c\left(C(\beta) \frac{55\left(1+3 \lambda^{-1}\right) \alpha+77(\alpha+\nu)}{20 \nu}+\frac{11 \sqrt{2} \alpha}{4 \lambda \nu \beta}+\frac{11 \sqrt{2}}{4 \beta}+1\right)\|f\|_{s-1} h^{s} \\
& +c\left(C^{2}(\beta) \frac{11 N\left(1+3 \lambda^{-1}\right)^{2}}{\nu^{2}}+\frac{22 N}{\lambda^{2} \nu^{2} \beta}\right)\|f\|_{s-1}^{2} h^{2 s}+\frac{11 \alpha^{2}}{4 \lambda \nu(\alpha+\nu) \beta}\|f\|_{-1} \\
& +\frac{33 \alpha^{2}}{25 \lambda^{2} \nu^{2} \beta}\|f\|_{-1}+\frac{11 \alpha}{4(\alpha+\nu) \beta}\left(\frac{5 N\|f\|_{-1}}{4(\alpha+\nu)^{2}}\right)^{2^{m}-1}\|f\|_{-1} \\
& +\frac{33}{25 \beta}\left(\frac{5 N\|f\|_{-1}}{4(\alpha+\nu)^{2}}\right)^{2^{m+1}-2}\|f\|_{-1} \\
\leq & c(\beta)\left(1+\frac{\alpha}{\nu}+\frac{\alpha}{\lambda \nu}\right)\|f\|_{s-1} h^{s}+c(\beta)\left(1+\frac{1}{\lambda}+\frac{1}{\lambda^{2}}\right) \frac{\|f\|_{s-1}^{2} h^{2 s}}{\|f\|_{-1}} \\
& +\frac{11 \alpha^{2}}{4 \lambda \nu(\alpha+\nu) \beta}\|f\|_{-1}+\frac{33 \alpha^{2}}{25 \lambda^{2} \nu^{2} \beta}\|f\|_{-1} \\
& +\frac{11}{4 \beta}\left(\frac{5 N}{4(\alpha+\nu)^{2}}\|f\|_{-1}\right)^{2^{m}-1}\|f\|_{-1} \\
& +\frac{33}{25 \beta}\left(\frac{5 N}{4(\alpha+\nu)^{2}}\|f\|_{-1}\right)^{2^{m+1}-2}\|f\|_{-1}, m \geq 0,1 \leq s \leq k, k=1,2,
\end{aligned}
$$

which completes the proof of Theorem 4.1.

Theorem 4.2. Assume that $(u, p)$ is the solution to the Navier-Stokes equations (2.3) and the conditions of Theorems 2.1, 2.2 and Lemma 4.2 hold. Then the solution $\left(u_{m h}^{1}, p_{m h}^{1}\right)$ defined by Algorithm 2 satisfies

$$
\begin{gathered}
\leq c(\beta)\left(\left(1+\frac{1}{\lambda}+\frac{1}{\lambda^{2}}\right)\|f\|_{s-1} h^{s}+\left(\frac{1}{\lambda}+\frac{1}{\lambda^{2}}+\frac{1}{\lambda^{3}}\right) \frac{\|f\|_{s-1}^{2}}{\|f\|_{-1}} h^{2 s}\right) \\
+\left(\frac{\alpha}{4 \lambda^{2} \nu}+\frac{\alpha}{\lambda^{3}(\alpha+\nu)}+\frac{1}{4 \lambda}\left(\frac{N}{(\alpha+\nu)^{2}}\|f\|_{-1}\right)^{m}\right)\|f\|_{-1}
\end{gathered}
$$




$$
+\frac{4}{\lambda}\left(\frac{N}{(\alpha+\nu)^{2}}\|f\|_{-1}\right)^{2 m}\|f\|_{-1}, m \geq 0,1 \leq s \leq k, k=1,2,
$$

(4.24) $\left\|p-p_{m h}^{1}\right\|_{0}$

$$
\begin{aligned}
\leq & c(\beta)\left(1+\frac{1}{\lambda}+\frac{\alpha}{\nu}+\frac{\alpha}{\lambda \nu}+\frac{\alpha}{\lambda^{2} \nu}\right)\|f\|_{s-1} h^{s} \\
& +c(\beta)\left(1+\frac{1}{\lambda}+\frac{1}{\lambda^{2}}+\frac{1}{\lambda^{3}}\right) \frac{\|f\|_{s-1}^{2}}{\|f\|_{-1}} h^{2 s}+\frac{(3+\lambda) \alpha^{2}}{\beta \lambda^{2} \nu(\alpha+\nu)}\|f\|_{-1} \\
& +\frac{(3+\lambda) \alpha}{\beta \lambda^{3} \nu}\|f\|_{-1}+\frac{3+\lambda}{\beta \lambda}\left(\frac{N}{(\alpha+\nu)^{2}}\|f\|_{-1}\right)^{m}\|f\|_{-1} \\
& +\frac{4(3+\lambda)}{\beta \lambda}\left(\frac{N}{(\alpha+\nu)^{2}}\|f\|_{-1}\right)^{2 m}\|f\|_{-1}, m \geq 0,1 \leq s \leq k, k=1,2 .
\end{aligned}
$$

Proof. It is noted that (4.17) also holds for Algorithm 2. From Theorem 3.1 and Lemma 4.2, we have

$$
\begin{aligned}
& \alpha+\nu-N \| \nabla u_{m h}^{0} \|_{0} \geq(\alpha+\nu)\left(1-\frac{N}{(\alpha+\nu)^{2}}\|f\|_{-1}\right)>\lambda(\alpha+\nu), \\
& \alpha+\nu+2 N\left\|\nabla u_{m h}^{0}\right\|_{0} \leq(\alpha+\nu)\left(1+\frac{2 N}{(\alpha+\nu)^{2}}\|f\|_{-1}\right)<3(\alpha+\nu), \\
& \alpha\left\|\nabla e^{0}\right\|_{0} \leq C(\beta)\left(1+3 \lambda^{-1}\right) \alpha \inf _{w_{h} \in X_{h}}\left\|\nabla\left(u-w_{h}\right)\right\|_{0} \\
&+\alpha \sqrt{2} \lambda^{-1} \nu^{-1} \inf _{q_{h} \in M_{h}}\left\|p-q_{h}\right\|_{0}+\lambda^{-1} \frac{\alpha^{2}}{\nu(\alpha+\nu)}\|f\|_{-1} \\
&+\frac{\alpha}{\alpha+\nu}\left(\frac{N}{(\alpha+\nu)^{2}}\|f\|_{-1}\right)^{m}\|f\|_{-1}, \quad m \geq 0 \\
& N\left\|\nabla e^{0}\right\|_{0}^{2} \leq C^{2}(\beta) 4 N\left(1+3 \lambda^{-1}\right)^{2}\left(\inf _{w_{h} \in X_{h}}\left\|\nabla\left(u-w_{h}\right)\right\|_{0}\right)^{2} \\
&+\frac{8 N}{\lambda^{2} \nu^{2}}\left(\inf _{q_{h} \in M_{h}}\left\|p-q_{h}\right\|_{0}\right)^{2}+\frac{4 \alpha^{2}}{\lambda^{2}(\alpha+\nu)^{2}}\|f\|_{-1} \\
&+4\left(\frac{N}{(\alpha+\nu)^{2}}\|f\|_{-1}\right)^{2 m}\|f\|_{-1}, m \geq 0
\end{aligned}
$$

Therefore, the triangle inequality, together with (4.17) and (4.25)-(4.28), implies

$$
\begin{aligned}
& \nu\left\|\nabla\left(u-u_{m h}^{1}\right)\right\|_{0} \\
\leq & C(\beta)\left(\frac{\left(1+3 \lambda^{-1}\right) \alpha}{\lambda(\alpha+\nu)}+3 \lambda^{-1}+1\right) \nu \inf _{w_{h} \in X_{h}}\left\|\nabla\left(u-w_{h}\right)\right\|_{0} \\
+ & \frac{\sqrt{2}\left(\lambda^{-1} \alpha+\nu\right)}{\lambda(\alpha+\nu)} \inf _{q_{h} \in M_{h}}\left\|p-q_{h}\right\|_{0}
\end{aligned}
$$




$$
\begin{aligned}
& +C^{2}(\beta) \frac{4 N\left(1+3 \lambda^{-1}\right)^{2} \nu}{\lambda(\alpha+\nu)}\left(\inf _{w_{h} \in X_{h}}\left\|\nabla\left(u-w_{h}\right)\right\|_{0}\right)^{2} \\
& +\frac{8 N}{\lambda^{3} \nu(\alpha+\nu)}\left(\inf _{q_{h} \in M_{h}}\left\|p-q_{h}\right\|_{0}\right)^{2}+\frac{\alpha^{2}}{\lambda^{2}(\alpha+\nu)^{2}}\|f\|_{-1} \\
& +\frac{4 \alpha^{2} \nu}{\lambda^{3}(\alpha+\nu)^{3}}\|f\|_{-1}+\frac{\nu \alpha}{\lambda(\alpha+\nu)^{2}}\left(\frac{N}{(\alpha+\nu)^{2}}\|f\|_{-1}\right)^{m}\|f\|_{-1} \\
& +\frac{4 \nu}{\lambda(\alpha+\nu)}\left(\frac{N}{(\alpha+\nu)^{2}}\|f\|_{-1}\right)^{2 m}\|f\|_{-1}, m \geq 0 .
\end{aligned}
$$

The applications of Theorem 2.2 and the uniqueness condition (2.5) lead to

$$
\begin{aligned}
& \nu\left\|\nabla\left(u-u_{m h}^{1}\right)\right\|_{0} \\
\leq & c(\beta)\left(1+\frac{1}{\lambda}+\frac{1}{\lambda^{2}}\right)\|f\|_{s-1} h^{s}+c(\beta)\left(\frac{1}{\lambda}+\frac{1}{\lambda^{2}}+\frac{1}{\lambda^{3}}\right) \frac{\|f\|_{s-1}^{2}}{\|f\|_{-1}} h^{2 s} \\
& +\frac{\alpha^{2}}{\lambda^{2}(\alpha+\nu)^{2}}\|f\|_{-1}+\frac{4 \alpha^{2} \nu}{\lambda^{3}(\alpha+\nu)^{3}}\|f\|_{-1} \\
& +\frac{\nu \alpha}{\lambda(\alpha+\nu)^{2}}\left(\frac{N}{(\alpha+\nu)^{2}}\|f\|_{-1}\right)^{m}\|f\|_{-1} \\
& +\frac{4 \nu}{\lambda(\alpha+\nu)}\left(\frac{N}{(\alpha+\nu)^{2}}\|f\|_{-1}\right)^{2 m}\|f\|_{-1}, m \geq 0,1 \leq s \leq k, k=1,2 .
\end{aligned}
$$

Using again the inequalities $(\alpha+\nu)^{2} \geq 4 \alpha \nu$ and $\frac{\nu}{\alpha+\nu}<1$, we get (4.23).

On the other hand, taking $q=0$ in (4.22) (noting that (4.22) is also valid for Algorithm 2) and from the inf-sup condition (2.9), (2.2), (4.8), the uniqueness condition (2.5), (4.26)-(4.29), we deduce that

$$
\begin{aligned}
& \beta\left\|q_{h}-p_{m h}^{1}\right\|_{0} \\
\leq & (\alpha+\nu)\left\|\nabla e^{1}\right\|_{0}+2 N\left\|\nabla e^{1}\right\|_{0}\left\|\nabla u_{m h}^{0}\right\|_{0}+\sqrt{2}\left\|p-q_{h}\right\|_{0}+N\left\|\nabla e^{0}\right\|_{0}^{2}+\alpha\left\|\nabla e^{0}\right\|_{0} \\
\leq & 3(\alpha+\nu)\left\|\nabla e^{1}\right\|_{0}+\sqrt{2}\left\|p-q_{h}\right\|_{0}+N\left\|\nabla e^{0}\right\|_{0}^{2}+\alpha\left\|\nabla e^{0}\right\|_{0} \\
\leq & C(\beta)\left(\frac{(3+\lambda)\left(1+3 \lambda^{-1}\right) \alpha+3(3+\lambda)(\alpha+\nu)}{\lambda}\right) \inf _{w_{h} \in X_{h}}\left\|\nabla\left(u-w_{h}\right)\right\|_{0} \\
& +\left(\frac{\left(1+3 \lambda^{-1}\right) \sqrt{2} \alpha}{\lambda \nu}+\frac{(3+\lambda) \sqrt{2}}{\lambda}\right) \inf _{q_{h} \in M_{h}}\left\|p-q_{h}\right\|_{0} \\
& +C^{2}(\beta) \frac{4 N(3+\lambda)\left(1+3 \lambda^{-1}\right)^{2}}{\lambda}\left(\inf _{w_{h} \in X_{h}}\left\|\nabla\left(u-w_{h}\right)\right\|_{0}\right)^{2} \\
& +\frac{8(3+\lambda) N}{\lambda^{3} \nu^{2}}\left(\inf _{q_{h} \in M_{h}}\left\|p-q_{h}\right\|_{0}\right)^{2}+\frac{(3+\lambda) \alpha^{2}}{\lambda^{2} \nu(\alpha+\nu)}\|f\|_{-1} \\
& +\frac{4(3+\lambda) \alpha^{2}}{\lambda^{3}(\alpha+\nu)^{2}}\|f\|_{-1}+\frac{(3+\lambda) \alpha}{\lambda(\alpha+\nu)}\left(\frac{N}{(\alpha+\nu)^{2}}\|f\|_{-1}\right)^{m}\|f\|_{-1}
\end{aligned}
$$




$$
+\frac{4(3+\lambda)}{\lambda}\left(\frac{N}{(\alpha+\nu)^{2}}\|f\|_{-1}\right)^{2 m}\|f\|_{-1}, m \geq 0 .
$$

Applying the triangle inequality, Theorem 2.2 and the uniqueness condition (2.5), we have

$$
\begin{aligned}
\leq & c(\beta)\left(1+\frac{1}{\lambda}+\frac{\alpha}{\nu}+\frac{\alpha}{\lambda \nu}+\frac{\alpha}{\lambda^{2} \nu}\right)\|f\|_{s-1} h^{s} \\
& +c(\beta)\left(1+\frac{1}{\lambda}+\frac{1}{\lambda^{2}}+\frac{1}{\lambda^{3}}\right) \frac{\|f\|_{s-1}^{2}}{\|f\|_{-1}} h^{2 s} \\
& +\frac{(3+\lambda) \alpha^{2}}{\beta \lambda^{2} \nu(\alpha+\nu)}\|f\|_{-1}+\frac{4(3+\lambda) \alpha^{2}}{\beta \lambda^{3}(\alpha+\nu)^{2}}\|f\|_{-1} \\
& +\frac{(3+\lambda) \alpha}{\beta \lambda(\alpha+\nu)}\left(\frac{N}{(\alpha+\nu)^{2}}\|f\|_{-1}\right)^{m}\|f\|_{-1} \\
& +\frac{4(3+\lambda)}{\beta \lambda}\left(\frac{N}{(\alpha+\nu)^{2}}\|f\|_{-1}\right)^{2 m}\|f\|_{-1}, m \geq 0,1 \leq s \leq k, k=1,2,
\end{aligned}
$$

which, together with the inequalities $(\alpha+\nu)^{2} \geq 4 \alpha \nu$ and $\frac{\alpha}{\alpha+\nu}<1$, finishes the proof of Theorem 4.2 .

In the estimators of Theorems 4.1-4.2, there is an error factor $\frac{N}{(\alpha+\nu)^{2}}\|f\|_{-1}$ related to the nonlinear iterations which is not easy to compute. Alternatively, we can derive the following error estimates by analogous arguments as those used for Theorems 4.1-4.2.

Theorem 4.3. Under the conditions of Theorems 2.1, 2.2 and Lemma 4.1, the solution $\left(u_{m h}^{1}, p_{m h}^{1}\right)$ defined by Algorithm 1 satisfies

$$
\begin{aligned}
& \nu\left\|\nabla\left(u-u_{m h}^{1}\right)\right\|_{0} \\
\leq & c(\beta)\left(1+\frac{1}{\lambda}\right)\|f\|_{s-1} h^{s}+c(\beta)\left(1+\frac{1}{\lambda}+\frac{1}{\lambda^{2}}\right) \frac{\|f\|_{s-1}^{2}}{\|f\|_{-1}} h^{2 s} \\
& +\frac{5 \alpha}{16 \lambda \nu}\|f\|_{-1}+\frac{3 \alpha^{2}}{5 \lambda^{2} \nu(\alpha+\nu)}\|f\|_{-1} \\
& +c|\log h|^{\frac{1}{2}}\left\|\nabla\left(u_{h 0}^{m}-u_{h 0}^{m-1}\right)\right\|_{0}\left\|u_{h 0}^{m}-u_{h 0}^{m-1}\right\|_{0} \\
& +\frac{c|\log h|}{\|f\|_{-1}}\left\|\nabla\left(u_{h 0}^{m}-u_{h 0}^{m-1}\right)\right\|_{0}^{2}\left\|u_{h 0}^{m}-u_{h 0}^{m-1}\right\|_{0}^{2}, m \geq 1,1 \leq s \leq k, k=1,2,
\end{aligned}
$$

$$
\left\|p-p_{m h}^{1}\right\|_{0}
$$




$$
\begin{aligned}
\leq & c(\beta)\left(1+\frac{\alpha}{\nu}+\frac{\alpha}{\lambda \nu}\right)\|f\|_{s-1} h^{s}+c(\beta)\left(1+\frac{1}{\lambda}+\frac{1}{\lambda^{2}}\right) \frac{\|f\|_{s-1}^{2}}{\|f\|_{-1}} h^{2 s} \\
& +\frac{11 \alpha^{2}}{4 \lambda \nu(\alpha+\nu) \beta}\|f\|_{-1}+\frac{33 \alpha^{2}}{25 \lambda^{2} \nu^{2} \beta}\|f\|_{-1} \\
& +c(\beta)|\log h|^{\frac{1}{2}}\left\|\nabla\left(u_{h 0}^{m}-u_{h 0}^{m-1}\right)\right\|_{0}\left\|u_{h 0}^{m}-u_{h 0}^{m-1}\right\|_{0} \\
& +\frac{c(\beta)|\log h|}{\|f\|_{-1}}\left\|\nabla\left(u_{h 0}^{m}-u_{h 0}^{m-1}\right)\right\|_{0}^{2}\left\|u_{h 0}^{m}-u_{h 0}^{m-1}\right\|_{0}^{2}, m \geq 1,1 \leq s \leq k, k=1,2 .
\end{aligned}
$$

Theorem 4.4. Assume that the conditions of Theorems 2.1, 2.2 and Lemma 4.2 hold. Then the solution $\left(u_{m h}^{1}, p_{m h}^{1}\right)$ obtained from Algorithm 2 satisfies

$$
\begin{aligned}
& \nu\left\|\nabla\left(u-u_{m h}^{1}\right)\right\|_{0} \\
\leq & c(\beta)\left(1+\frac{1}{\lambda}+\frac{1}{\lambda^{2}}\right)\|f\|_{s-1} h^{s}+c(\beta)\left(\frac{1}{\lambda}+\frac{1}{\lambda^{2}}+\frac{1}{\lambda^{3}}\right) \frac{\|f\|_{s-1}^{2}}{\|f\|_{-1}} h^{2 s} \\
& +\frac{\alpha}{4 \lambda^{2} \nu}\|f\|_{-1}+\frac{\alpha}{\lambda^{3}(\alpha+\nu)}\|f\|_{-1}+\frac{c}{\lambda} \nu\left\|u_{h 0}^{m}-u_{h 0}^{m-1}\right\|_{0} \\
& +\frac{c}{\lambda\|f\|_{-1}} \nu^{2}\left\|u_{h 0}^{m}-u_{h 0}^{m-1}\right\|_{0}^{2}, m \geq 1,1 \leq s \leq k, k=1,2,
\end{aligned}
$$

$$
\begin{aligned}
& \left\|p-p_{m h}^{1}\right\|_{0} \\
\leq & c(\beta)\left(1+\frac{1}{\lambda}+\frac{\alpha}{\nu}+\frac{\alpha}{\lambda \nu}+\frac{\alpha}{\lambda^{2} \nu}\right)\|f\|_{s-1} h^{s} \\
& +c(\beta)\left(1+\frac{1}{\lambda}+\frac{1}{\lambda^{2}}+\frac{1}{\lambda^{3}}\right) \frac{\|f\|_{s-1}^{2}}{\|f\|_{-1}} h^{2 s} \\
& +\frac{(3+\lambda) \alpha^{2}}{\beta \lambda^{2} \nu(\alpha+\nu)}\|f\|_{-1}+\frac{(3+\lambda) \alpha}{\beta \lambda^{3} \nu}\|f\|_{-1}+\frac{c(1+\lambda) \alpha}{\beta \lambda}\left\|u_{h 0}^{m}-u_{h 0}^{m-1}\right\|_{0} \\
& +\frac{c(1+\lambda)}{\beta \lambda\|f\|_{-1}} \nu^{2}\left\|u_{h 0}^{m}-u_{h 0}^{m-1}\right\|_{0}^{2}, \quad m \geq 1,1 \leq s \leq k, k=1,2 .
\end{aligned}
$$

Remark 4.4. In the estimators (4.31) and (4.33), the last two terms related to the nonlinear iterations not only indicate the contributions of the nonlinear iterations (or linearizations) to error of the approximate solution, but also suggest a suitable stopping criterion for the nonlinear iterations in the defect step, i.e.,

$$
\nu^{-1}|\log h|^{\frac{1}{2}}\left\|\nabla\left(u_{h 0}^{n}-u_{h 0}^{n-1}\right)\right\|_{0}\left\|u_{h 0}^{n}-u_{h 0}^{n-1}\right\|_{0}<c h^{s}
$$

for Algorithm 1 and

$$
\left\|u_{h 0}^{n}-u_{h 0}^{n-1}\right\|_{0}<c h^{s}
$$

for Algorithm 2 (noting that $\|f\|_{-1}^{-1}|\log h|\left\|\nabla\left(u_{h 0}^{n}-u_{h 0}^{n-1}\right)\right\|_{0}^{2}\left\|u_{h 0}^{n}-u_{h 0}^{n-1}\right\|_{0}^{2}$ is typically a higher order term compared to

$$
|\log h|^{\frac{1}{2}}\left\|\nabla\left(u_{h 0}^{n}-u_{h 0}^{n-1}\right)\right\|_{0}\left\|u_{h 0}^{n}-u_{h 0}^{n-1}\right\|_{0},
$$


and $\nu\left\|u_{h 0}^{n}-u_{h 0}^{n-1}\right\|_{0}^{2}$ is usually a higher order term compared to

$$
\nu^{2}\|f\|_{-1}^{-1}\left\|u_{h 0}^{n}-u_{h 0}^{n-1}\right\|_{0}
$$

as $n$ tends to infinity). This is a very useful tool in practical calculations. In the next section, we shall give some numerical results to support this observation.

Remark 4.5. For the $j$-step corrected solution $(j \geq 2)$, it is cumbersome to derive the explicit dependence of the errors upon the parameters such as $\nu, \alpha$ and $\lambda$. Define

$$
\lambda_{j}=\alpha+\nu-N\left\|\nabla u_{m h}^{j}\right\|_{0} .
$$

Under the condition $\lambda_{j-1}>0$ and using an analogous argument for Theorems 4.1-4.2, we can get the following result:

$$
\begin{aligned}
& \left\|\nabla\left(u-u_{m h}^{j}\right)\right\|_{0} \\
\leq & C(\beta)\left(1+\frac{\alpha+\nu+2 N\left\|\nabla u_{m h}^{j-1}\right\|_{0}}{\lambda_{j-1}}\right) \inf _{w_{h} \in X_{h}}\left\|\nabla\left(u-w_{h}\right)\right\|_{0} \\
& +\frac{1}{\lambda_{j-1}}\left(\sqrt{2} \inf _{q_{h} \in M_{h}}\left\|p-q_{h}\right\|_{0}+N\left\|\nabla e^{j-1}\right\|_{0}^{2}+\alpha\left\|\nabla e^{j-1}\right\|_{0}\right) \\
\leq & C(\beta)\left(1+\frac{\alpha+\nu+2 N\left\|\nabla u_{m h}^{j-1}\right\|_{0}}{\lambda_{j-1}}\right) \nu^{-1}\|f\|_{s-1} h^{s} \\
& +\frac{1}{\lambda_{j-1}}\left(c \sqrt{2}\|f\|_{s-1} h^{s}+N\left\|\nabla e^{j-1}\right\|_{0}^{2}+\alpha\left\|\nabla e^{j-1}\right\|_{0}\right), 1 \leq s \leq k, k=1,2 .
\end{aligned}
$$

Therefore, provided that $u_{m h}^{j-1}$ is close enough to $u$ (and hence $\lambda_{j-1}>0$ ) and noting that $\left\|\nabla e^{j-1}\right\|_{0}^{2}$ is typically a higher order term compared to $\left\|\nabla e^{j-1}\right\|_{0}$, by a heuristic analysis, we can see that each correction step improves the error in the previous step by one power of $h$ (recall that typically $\alpha=\mathcal{O}(h)$ ) in the first $s$ steps corrections. However, noting that there is a factor $\lambda^{-1}$ in the error $\left\|\nabla e^{1}\right\|_{0}$ (see Theorems 4.1-4.2), and consequently, $\left(\lambda \prod_{j=1}^{s-1} \lambda_{j}\right)^{-1}$ in the error $\left\|\nabla e^{s}\right\|_{0}$ of the $s$-step corrected solution, to ensure that a good approximate solution can be obtained by the defect-correction algorithms in cases of small $\lambda$ (see (2.5) for the definition of $\lambda$ ), a little more than s steps corrections may be necessary, where $s$ is the degree of local polynomials in the finite element subspace $X_{h}$ of velocity.

Remark 4.6. From (4.38) we can see that each correction step improves the error in the previous step by one power of $\alpha$. Therefore, for those high Reynolds number flows where the stabilization parameter $\alpha$ needs to be chosen as very big (say, $1>\alpha>>h$ ) to make the stability conditions (4.1) or (4.7) hold for our algorithms, we can perform more correction steps to obtain a good 
approximate solution. The bigger the stabilization parameter $\alpha$, the more the correction steps.

\section{Numerical results}

In this section, we shall present some numerical results to verify the theoretical results and demonstrate the effectiveness of the proposed algorithms. A simple problem with known analytical solution is first tested to verify the theoretical results, and then two benchmark fluid flow problems, namely, the lid-driven cavity flow and the flow over a backward-facing step are simulated to illustrate the effectiveness of the proposed algorithms.

\subsection{Analytical solution}

In this test case, $\Omega$ is the unit square $[0,1] \times[0,1]$. The body force $f$ and the boundary conditions are derived from the exact solution to the steady NavierStokes equations given by

$$
\begin{aligned}
u_{1} & =10 x^{2}(x-1)^{2} y(y-1)(2 y-1), \\
u_{2} & =-10 y^{2}(y-1)^{2} x(x-1)(2 x-1), \\
p & =10(2 x-1)(2 y-1) .
\end{aligned}
$$

The uniform mesh consists of triangular elements which are obtained by dividing $\Omega$ into sub-squares of equal size and then drawing the diagonal in each sub-square.

TABLE 1. Errors of the approximate solutions computed with Mini elements $(\nu=0.1, \alpha=h)$.

\begin{tabular}{lllllr}
\hline Method & $h$ & $\frac{\left\|\nabla\left(u-u_{m h}^{1}\right)\right\|_{0}}{\|\nabla u\|_{0}}$ & $\frac{\left\|p-p_{m h}^{1}\right\|_{0}}{\|p\|_{0}}$ & $u_{H^{1}}$ rate & $p_{L^{2}}$ rate \\
\hline Algorithm 1 & $1 / 25$ & 0.171702 & 0.00127869 & - & - \\
& $1 / 36$ & 0.10256 & 0.00062198 & 1.41318 & 1.97642 \\
& $1 / 49$ & 0.0677923 & 0.000339585 & 1.34285 & 1.96296 \\
& $1 / 64$ & 0.0482832 & 0.000201724 & 1.27073 & 1.95019 \\
& $1 / 81$ & 0.0363272 & 0.000127808 & 1.2078 & 1.93733 \\
Method of $[26]$ & $1 / 100$ & 0.0284642 & $8.52064 \mathrm{e}-005$ & 1.15755 & 1.92413 \\
& $1 / 25$ & 0.171707 & 0.00128182 & - & - \\
& $1 / 36$ & 0.102563 & 0.000626125 & 1.41319 & 1.9649 \\
& $1 / 49$ & 0.0677939 & 0.000344372 & 1.34285 & 1.9391 \\
& $1 / 64$ & 0.0482844 & 0.000206876 & 1.27073 & 1.90818 \\
& $1 / 81$ & 0.0363281 & 0.000133135 & 1.2078 & 1.87104 \\
& $1 / 100$ & 0.028465 & $9.05813 \mathrm{e}-005$ & 1.15754 & 1.82762 \\
\hline
\end{tabular}

Firstly, to test the asymptotical errors provided by our algorithms with respect to the mesh parameter $h$, we set $\nu=0.1, \alpha=h$ and compute the finite element solutions by Algorithms 1-2 and the method of [26] on meshes 
TABlE 2. Errors of the approximate solutions computed with Taylor-Hood elements $(\nu=0.1, \alpha=h)$.

\begin{tabular}{lllllr}
\hline Method & $h$ & $\frac{\left\|\nabla\left(u-u_{m h}^{2}\right)\right\|_{0}}{\|\nabla v u\|_{0}}$ & $\frac{\left\|p-p_{m h}^{2}\right\|_{0}}{\|p\|_{0}}$ & $u_{H^{1}}$ rate & $p_{L^{2}}$ rate \\
\hline Algorithm 1 & $1 / 25$ & 0.0237932 & 0.00123946 & - & - \\
& $1 / 36$ & 0.0105223 & 0.000597723 & 2.23755 & 2.00004 \\
& $1 / 49$ & 0.0050216 & 0.000322631 & 2.39942 & 2.00005 \\
& $1 / 64$ & 0.00257075 & 0.000189118 & 2.50709 & 2.00005 \\
& $1 / 81$ & 0.00140117 & 0.000118064 & 2.57632 & 2.00004 \\
Method of $[26]$ & $1 / 100$ & 0.000807201 & $7.74613 \mathrm{e}-005$ & 2.61715 & 2.00003 \\
& $1 / 25$ & 0.0237949 & 0.00123979 & - & - \\
& $1 / 36$ & 0.0105238 & 0.00059796 & 2.23734 & 1.99969 \\
& $1 / 49$ & 0.00502293 & 0.000322791 & 2.39904 & 1.99973 \\
& $1 / 64$ & 0.00257186 & 0.000189226 & 2.50646 & 1.99977 \\
& $1 / 81$ & 0.00140209 & 0.000118138 & 2.57536 & 1.99981 \\
& $1 / 100$ & 0.000807967 & $7.7513 \mathrm{e}-005$ & 2.61577 & 1.99984 \\
\hline
\end{tabular}

TABLE 3. Errors of the approximate solutions computed with different values of viscosity (Mini elements, $\alpha=h$ ).

\begin{tabular}{|c|c|c|c|c|c|c|}
\hline \multirow[t]{2}{*}{$\nu$} & \multicolumn{2}{|c|}{ Algorithm 1} & \multicolumn{2}{|c|}{ Algorithm 2} & \multicolumn{2}{|c|}{ Method of [26] } \\
\hline & $\frac{\left\|\nabla\left(u-u_{m h}^{\perp}\right)\right\|_{0}}{\|\nabla u\|_{0}}$ & $\frac{\left\|p-p_{m h}^{\perp}\right\|_{0}}{\|p\|_{0}}$ & $\frac{\left\|\nabla\left(u-u_{m h}^{\perp}\right)\right\|_{0}}{\|\nabla u\|_{0}}$ & $\frac{\left\|p-p_{m h}^{\perp}\right\|_{0}}{\|p\|_{0}}$ & $\frac{\left\|\nabla\left(u-u_{m h}^{\perp}\right)\right\|_{0}}{\|\nabla u\|_{0}}$ & $\frac{\left\|p-p_{m h}^{\perp}\right\|_{0}}{\|p\|_{0}}$ \\
\hline 0.1 & 0.0284642 & $8.52064 \mathrm{e}-005$ & 0.0284642 & $8.52064 \mathrm{e}-005$ & 0.028465 & $9.05813 \mathrm{e}-005$ \\
\hline 0.01 & 0.257386 & 0.000177475 & 0.257386 & 0.000177476 & 0.257453 & 0.000214019 \\
\hline 0.001 & 0.837118 & 0.000321053 & 0.837117 & 0.000321053 & 0.837155 & 0.000323375 \\
\hline 0.0001 & 0.99192 & 0.00032818 & 0.99192 & 0.00032818 & 0.991924 & 0.000328204 \\
\hline
\end{tabular}

TABLE 4. Errors of the approximate solutions computed with different values of viscosity (Taylor-Hood elements, $\alpha=h$ ).

\begin{tabular}{|c|c|c|c|c|c|c|}
\hline \multirow[t]{2}{*}{$\nu$} & \multicolumn{2}{|c|}{ Algorithm 1} & \multicolumn{2}{|c|}{ Algorithm 2} & \multicolumn{2}{|c|}{ Method of [26] } \\
\hline & $\frac{\left\|\nabla\left(u-u_{m h}^{2}\right)\right\|_{0}}{\|\nabla u\|_{0}}$ & $\frac{\left\|p-p_{m h}^{2}\right\|_{0}}{\|p\|_{0}}$ & $\frac{\left\|\nabla\left(u-u_{m h}^{2}\right)\right\|_{0}}{\|\nabla u\|_{0}}$ & $\frac{\left\|p-p_{m h}^{2}\right\|_{0}}{\|p\|_{0}}$ & $\frac{\left\|\nabla\left(u-u_{m h}^{2}\right)\right\|_{0}}{\|\nabla u\|_{0}}$ & $\frac{\left\|p-p_{m h}^{2}\right\|_{0}}{\|p\|_{0}}$ \\
\hline 0.1 & 0.000807201 & $7.74613 \mathrm{e}-005$ & 0.000807201 & $7.74613 \mathrm{e}-005$ & 0.000807967 & $7.7513 \mathrm{e}-005$ \\
\hline 0.01 & 0.125099 & 0.000111136 & 0.125099 & 0.000111136 & 0.125216 & 0.000134219 \\
\hline 0.001 & 0.752762 & 0.000310857 & 0.752762 & 0.000310857 & 0.75287 & 0.000314894 \\
\hline 0.0001 & 0.972607 & 0.000328058 & 0.972607 & 0.000328058 & 0.97262 & 0.000328106 \\
\hline
\end{tabular}

with size $h=\frac{1}{16}, \frac{1}{25}, \ldots, \frac{1}{100}$. It is noted that this laminar case does not require defect-correction method. We just use this simple example to verify the theoretical results. Both the Mini elements and Taylor-Hood elements are used for the finite element discretization. For Mini elements, the theory predicts a convergence rate of $\mathcal{O}(h)$ in energy norm (i.e., $H^{1}$-norm for the velocity and $L^{2}$-norm for the pressure), while that of Taylor-Hood elements is $\mathcal{O}\left(h^{2}\right)$. Therefore, according to our theoretical predictions, we perform one and 
TABlE 5. Number of nonlinear iterations in the defect step for the lid-driven cavity flow at various Reynolds numbers.

\begin{tabular}{lcccr}
\hline Method & $R e=1000$ & $R e=5000$ & $R e=7500$ & $R e=10000$ \\
\hline Algorithm 1 & 5 & 7 & 4 & 5 \\
Algorithm 2 & 17 & 30 & 40 & 52 \\
\hline
\end{tabular}
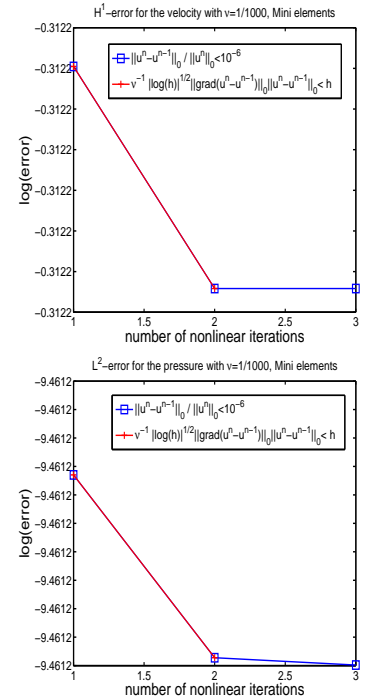

(a) $\nu=1 / 1000$
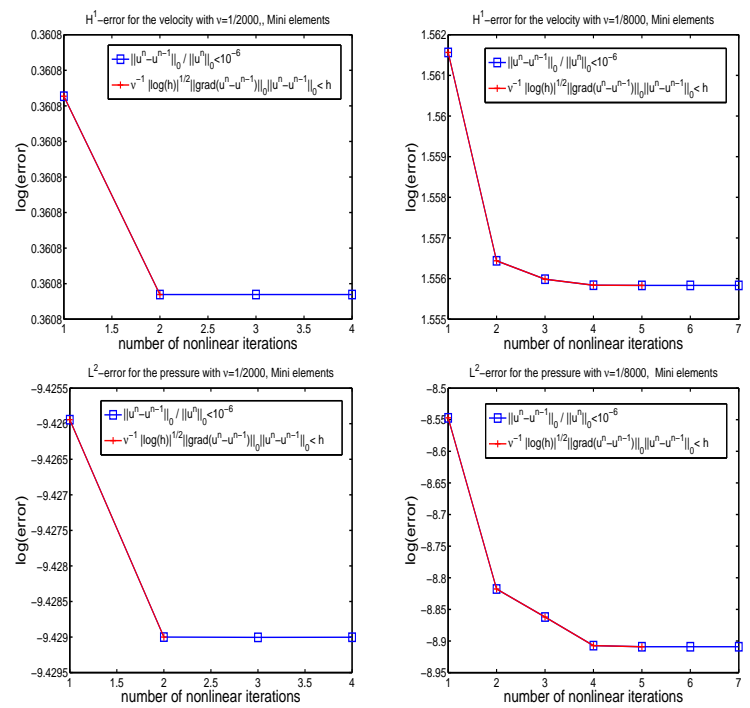

(b) $\nu=1 / 2000$

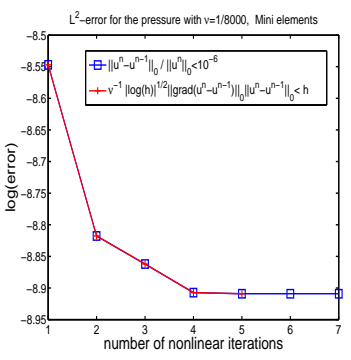

(c) $\nu=1 / 8000$

FiguRE 1. Evolution of the $H^{1}$-error for the velocity (top) and $L^{2}$-error for the pressure (bottom) with the nonlinear iterations (Algorithm 1, Mini elements, $h=1 / 100, \alpha=0.01 h$ ).

two-step corrections for Mini elements and Taylor-Hood elements, respectively. The public domain software FreeFem $++[14]$ is used. Motivated by Remark 4.4 , we employ the following stopping criteria for the nonlinear iterations in the defect step:

$$
\nu^{-1}|\log h|^{\frac{1}{2}}\left\|\nabla\left(u_{h 0}^{n}-u_{h 0}^{n-1}\right)\right\|_{0}\left\|u_{h 0}^{n}-u_{h 0}^{n-1}\right\|_{0}<h^{s}
$$

for Algorithm 1 and

$$
\left\|u_{h 0}^{n}-u_{h 0}^{n-1}\right\|_{0}<0.05 h^{s}
$$

for Algorithm 2, where $s=1$ for Mini elements and $s=2$ for Taylor-Hood elements.

In this test case, the numerical results show that there is no difference in errors of the computed solutions between Algorithms 1 and 2. Hence we just 

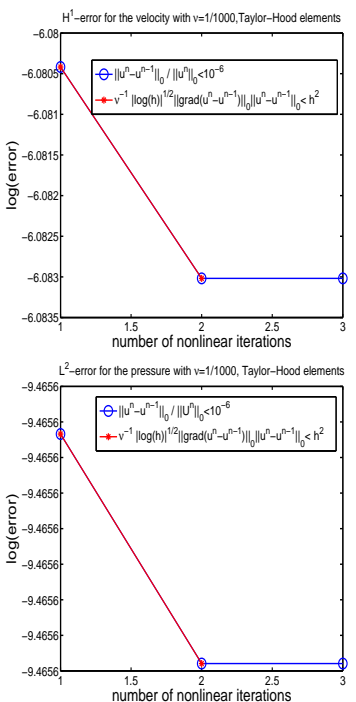

(a) $\nu=1 / 1000$
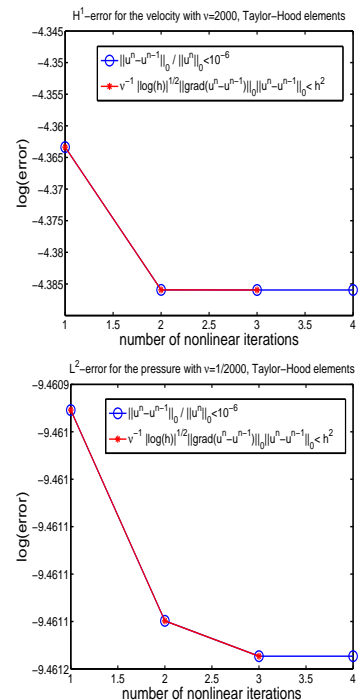

(b) $\nu=1 / 2000$
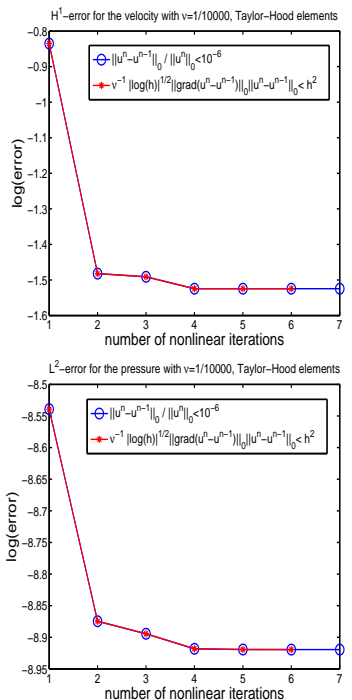

(c) $\nu=1 / 10000$

Figure 2. Evolution of the $H^{1}$-error for the velocity (top) and $L^{2}$-error for the pressure (bottom) with the nonlinear iterations (Algorithm 1, Taylor-Hood elements, $h=1 / 100, \alpha=$ $0.015 h)$.

present the computed results by Algorithm 1. In Tables 1-2, the numerical results computed with Mini elements and Taylor-Hood elements are listed, respectively. From Tables 1-2, we can see that the numerical results support the theoretical predictions well. Actually, for the velocity both with Mini elements and Taylor-Hood elements and for the pressure with Mini elements, this numerical example shows a better order of convergence than our theory predicted. While compared to the method of [26], the numerical results show that our method is better which yields a higher accurate solution (see Tables 1 and 2).

To further compare our method with the method of [26], we set $h=1 / 100$, $\alpha=h$ and compute the finite element solutions with different values of viscosity $\nu$ by our Algorithms 1-2 and the method of [26]. The numerical results listed in Tables 3 and 4 show that our method is better than the method of [26]; by our method, a better solution can be obtained.

As mentioned in Remark 4.4, the last two terms in the estimators (4.31) and (4.33) not only indicate how many contributions of the nonlinear iterations are made to error of the approximate solution, but also suggest a stopping criterion for the nonlinear iterations in the defect step. To attest this, we set $h=1 / 100$ and compute the finite element solution by Algorithms 1-2 with different values of $\nu$, different stability factor $\alpha$ and different finite elements pairs. For the sake 

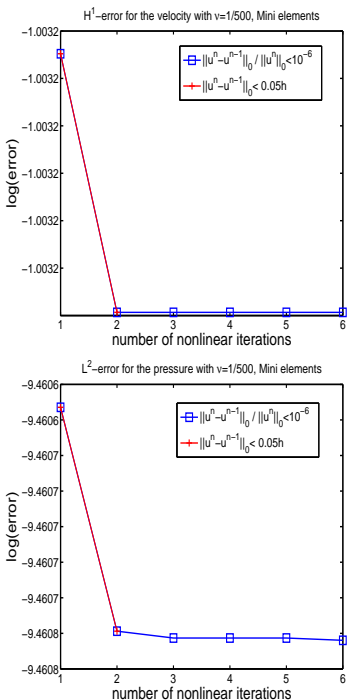

(a) $\nu=1 / 500$
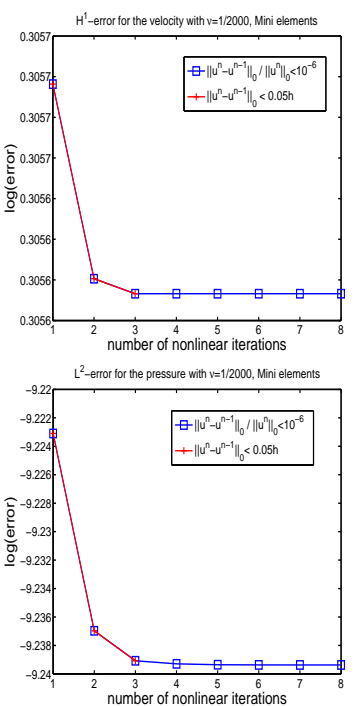

(b) $\nu=1 / 2000$
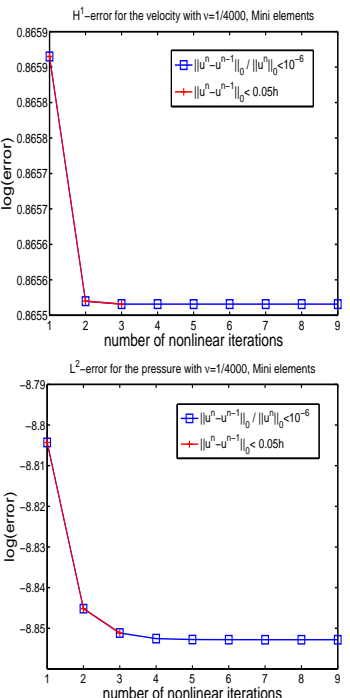

(c) $\nu=1 / 4000$

FIGURE 3. Evolution of the $H^{1}$-error for the velocity (top) and $L^{2}$-error for the pressure (bottom) with the nonlinear iterations (Algorithm 2, Mini elements, $h=1 / 100, \alpha=0.02 h$ ).

of comparison, we also employ a commonly used stopping criterion, i.e.,

$$
\frac{\left\|u_{h 0}^{n}-u_{h 0}^{n-1}\right\|_{0}}{\left\|u_{h 0}^{n}\right\|_{0}}<10^{-6} .
$$

Figures 1-4 depict the evolution of the relative errors with the nonlinear iterations. It is of worth to mention that the constant labels of the $y$-axis in some sub-figures of Figures (1)-(4) are due to the fact that only four decimal digits are shown. From Figures 1-4 we can see that, at the beginning of the nonlinear iterations, the errors of the approximate solutions decrease sharply with the nonlinear iterations; however, after a certain number of steps, further iterating does not lead to apparent decrease in errors of the computed solutions. A possible explanation is that, in the starting nonlinear iterations, the summation of the last two terms in the estimators (4.31)-(4.34) is dominant, leading to a decrease in errors of the approximate solutions as the values of these two terms drop (in general, the values of these two terms decrease with the nonlinear iterations provided that the iterative method used is convergent); while after a certain number of steps, the last two terms become smaller than the summation of the first four terms, leading to a smaller contribution to the errors of the solutions. What is more, when the last two terms become small 

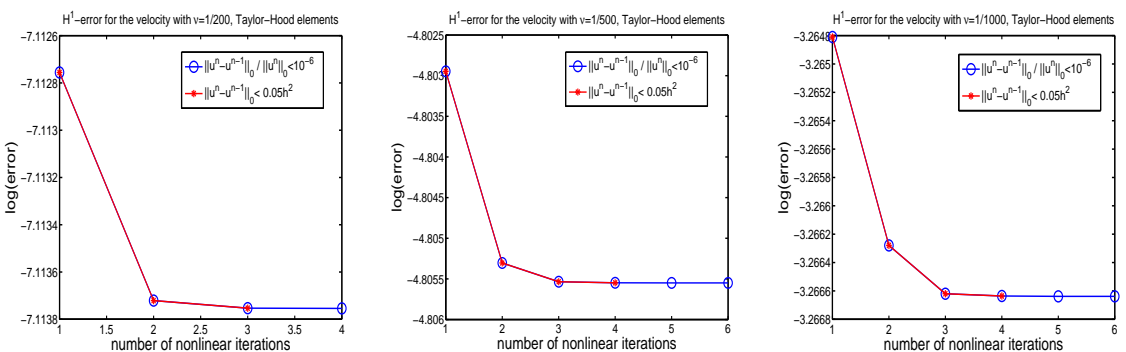

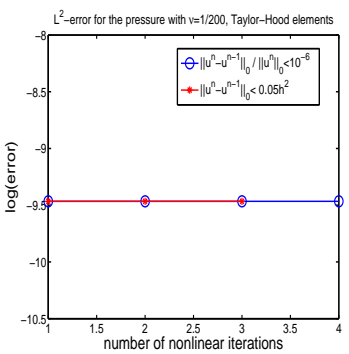

(a) $\nu=1 / 200$

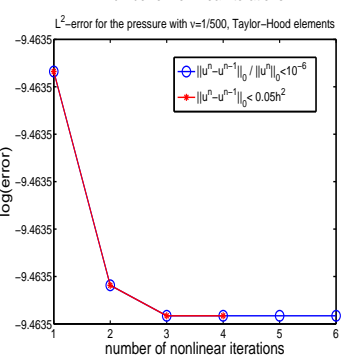

(b) $\nu=1 / 500$

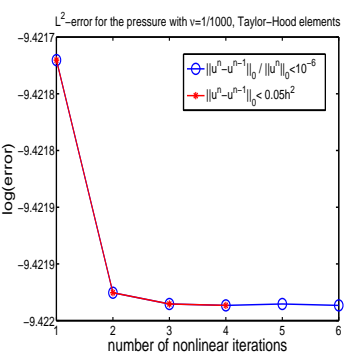

(c) $\nu=1 / 1000$

FiguRE 4. Evolution of the $H^{1}$-error for the velocity (top) and $L^{2}$-error for the pressure (bottom) with the nonlinear iterations (Algorithm 2, Taylor-Hood elements, $h=1 / 100, \alpha=$ $0.05 h)$.

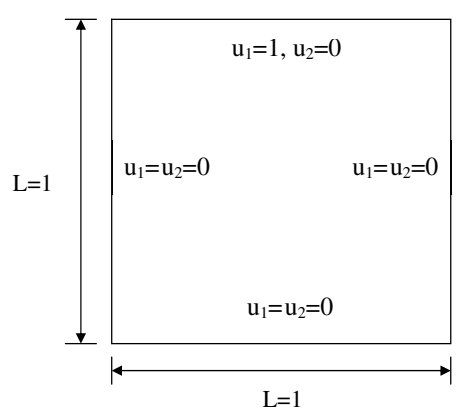

Figure 5. Schematic diagram of the lid-driven cavity flow.

to the extent that they can be neglected compared to the summation of the first four terms, preserving the accuracy of the approximate solutions will be observed. From these numerical results, we can see that a suitable stopping criterion for the nonlinear iterations is of great importance in practical calculations which may avoid redundant computations. This test case confirms our 

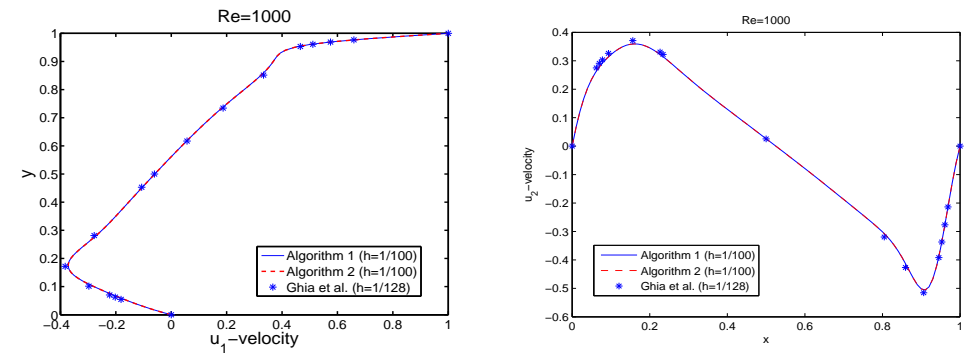

Figure 6 . Comparison of $u_{1}$-velocity profiles along the vertical centerline (left) and $u_{2}$-velocity profiles along the horizontal centerline (right) for lid-driven cavity flow at $R e=1000$.
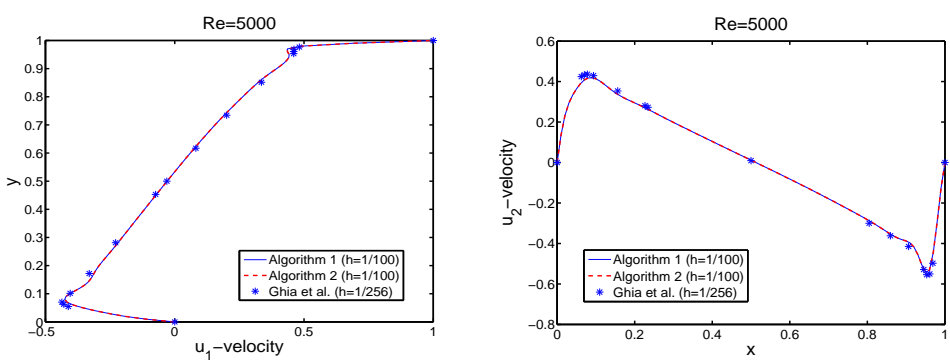

Figure 7 . Comparison of $u_{1}$-velocity profiles along the vertical centerline (left) and $u_{2}$-velocity profiles along the horizontal centerline (right) for lid-driven cavity flow at $R e=5000$.
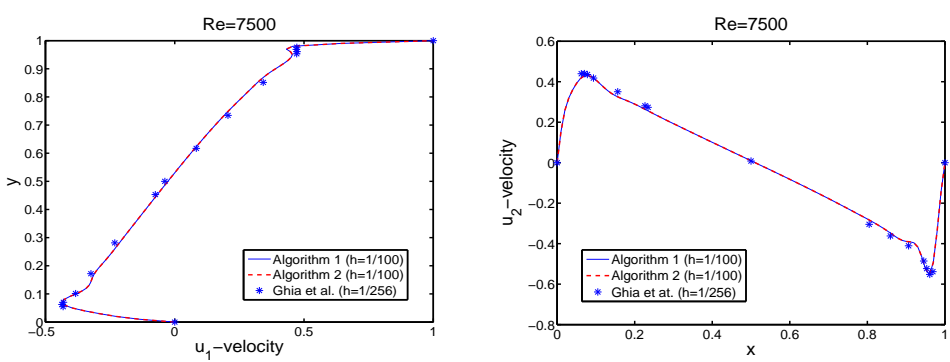

Figure 8 . Comparison of $u_{1}$-velocity profiles along the vertical centerline (left) and $u_{2}$-velocity profiles along the horizontal centerline (right) for lid-driven cavity flow at $R e=7500$.

theoretical results and shows that the stopping criteria (4.35) and (4.36) we derived are suitable and reliable. 

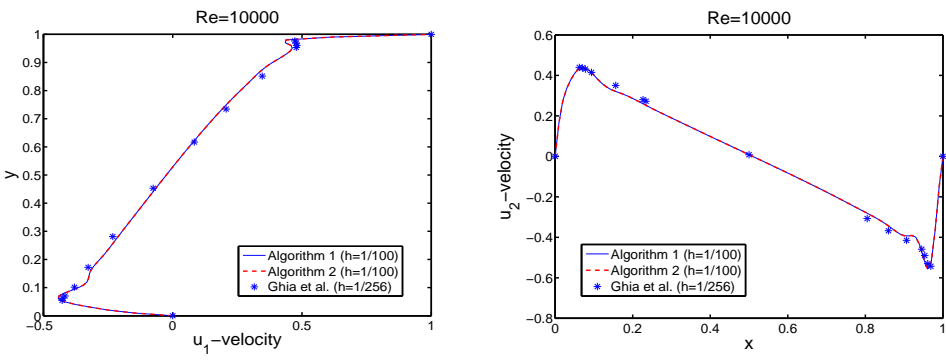

FiguRE 9. Comparison of $u_{1}$-velocity profiles along the vertical centerline (left) and $u_{2}$-velocity profiles along the horizontal centerline (right) for lid-driven cavity flow at $R e=10000$.
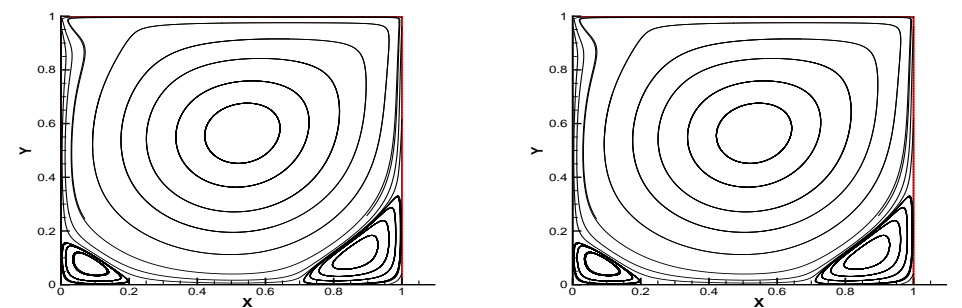

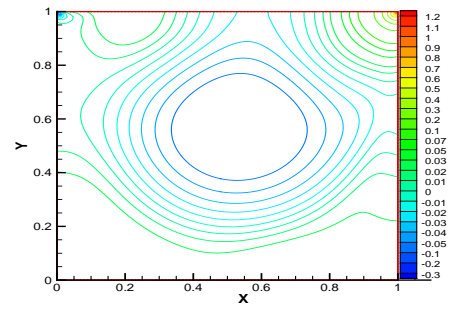

(a) Algorithm 1

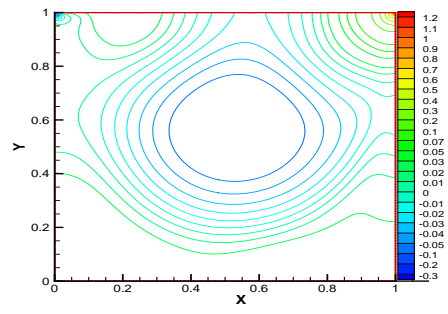

(b) Algorithm 2

Figure 10. Numerical streamlines (top) and isobars (bottom) for lid-driven cavity flow at $R e=1000$ : (a) Algorithm 1 and (b) Algorithm 2.

\subsection{Lid-driven cavity flow}

The second example we consider here is the incompressible lid-driven cavity flow problem defined in the unit square, which was investigated by many researchers. In this problem, velocities are zero on all edges except the top one (the lid), which has a driving horizontal velocity of one; see Figure 5 for schematic diagram. The Reynolds number for this problem is defined as 

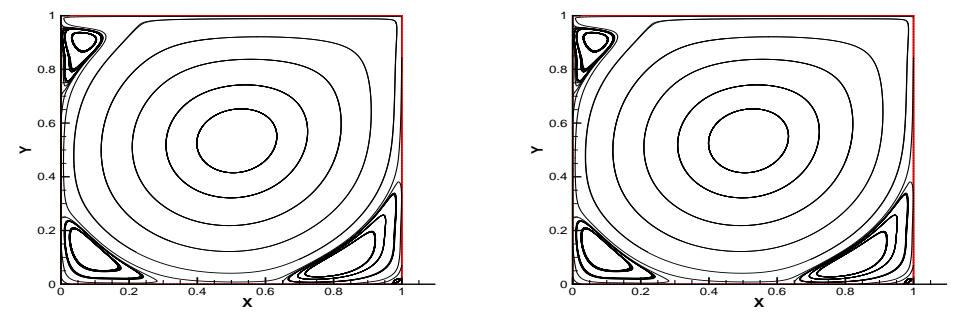

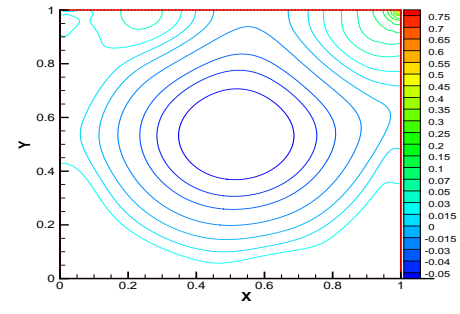

(a) Algorithm 1

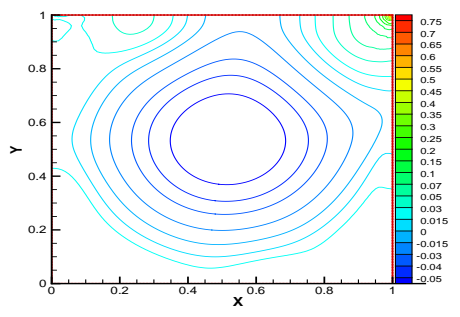

(b) Algorithm 2

FiguRE 11. Numerical streamlines (top) and isobars (bottom) for lid-driven cavity flow at $R e=5000$ : (a) Algorithm 1 and (b) Algorithm 2.

$R e=U L / \nu$, where $U$ (here $U=1$ ) is the velocity of the top lid and $L$ (here $L=1$ ) is the length of the side wall.

We compute an approximate solution at $R e=1000,5000,7500$ and 10000 on a uniform mesh with size $h=1 / 100$, and with the optimal artificial viscosity parameter $\alpha=0.02 h$, which was found by various precomputations. The Taylor-Hood elements are used. It is well known that the Newton iterative method for nonlinear system is sensitive to the initial guess, i.e., to ensure the convergence of the Newton iterations, the initial guess for the iterations should be close enough to the solution. Therefore, we compute a solution at $R e=500$ as the initial guess for $R e=1000$, while the computed solution at $R e=1000$ is used as the initial guess for $R e=5000$, and so on. The stopping criteria (5.2) and (5.3) are employed for Algorithms 1 and 2, respectively.

We compute the $x$ component of velocity along the vertical centerline and $y$ component of velocity along the horizontal centerline by Algorithms 1 and 2, and compare our numerical results with those of Ghia, Ghia and Shin [7] where the computations were based on the vortcity-stream function form of the Navier-Stokes equations on much finer $129 \times 129$ (for $R e=1000$ ) and $257 \times 257$ (for $R e=5000,7500,10000$ ) grid meshes. Figures 6-9 show that the accuracy of our computed solutions are very comparable to those of [7] although much coarser grid is used. Of interest is that there is no obvious difference in the 

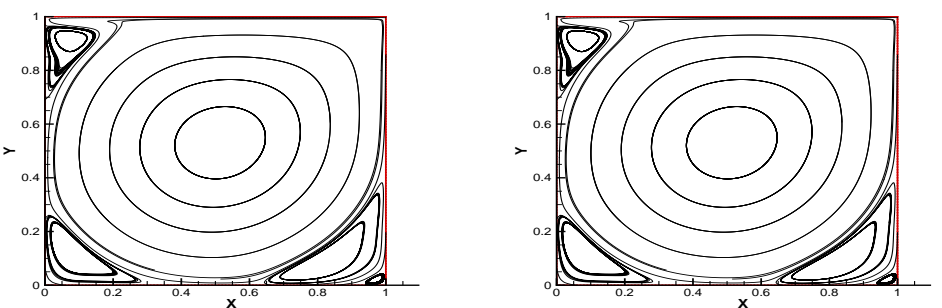

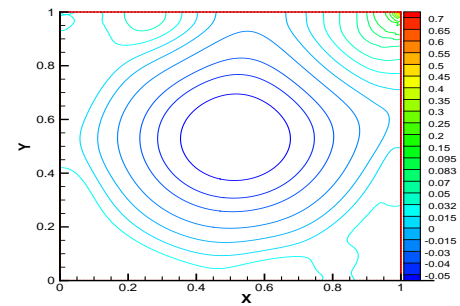

(a) Algorithm 1

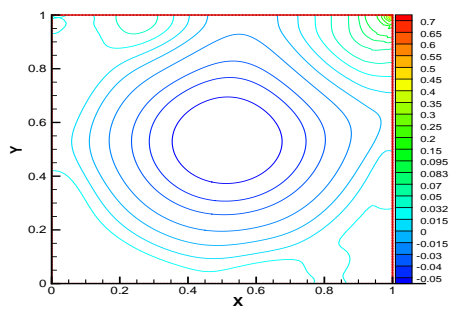

(b) Algorithm 2

FiguRE 12. Numerical streamlines (top) and isobars (bottom) for lid-driven cavity flow at $R e=7500$ : (a) Algorithm 1 and (b) Algorithm 2.

computed solutions between Algorithm 1 and Algorithm 2 although the number of nonlinear iterations in the defect step differs largely from each other, where Algorithm 1 converges much faster than Algorithm 2 (see Table 5 for details). Figures 10-13 describe the computed streamlines and isobars, which are in good agreement with those of [7].

\subsection{Flow over a backward-facing step}

In this example, we consider the $2 \mathrm{D}$ backward-facing step flow which is an important test problem for validating the robustness of a Navier-Stokes solver. The problem we consider here is defined on a long channel $[0,30] \times$ $[-0.5,0.5]$, with no-slip conditions imposed on the top and bottom walls, as well as the lower half part of the left wall. At the inlet boundary, a fully developed parabolic velocity profile $u_{1}=24 y(0.5-y)$ for $0 \leq y \leq 0.5$ is specified, which leads to a maximum inflow velocity of $u_{\max }=1.5$ and an average inflow velocity of $u_{\text {ave }}=1.0$. The outlet boundary condition is set as the free stream condition $-p+\nu \frac{\partial u_{1}}{\partial x}=0$. See Figure 14 for detailed geometry and boundary conditions information. The Reynolds number for this problem is defined as $R e=U_{\text {ave }} L / \nu$, where $U_{\text {ave }}(=1)$ is the average velocity at the inlet boundary and $L(=1)$ is the channel height. 

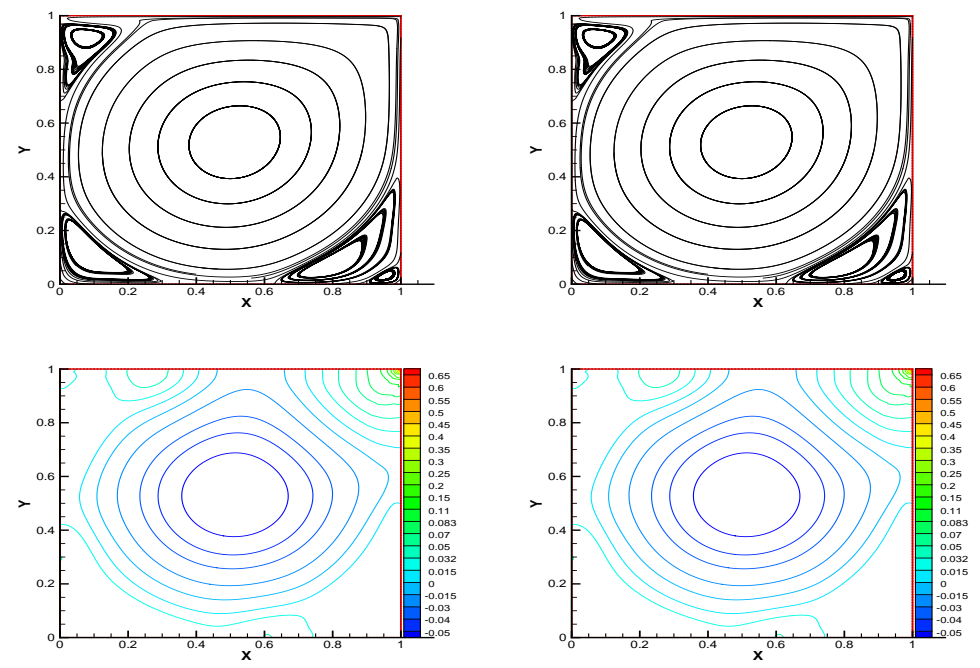

(a) Algorithm 1

(b) Algorithm 2

FiguRE 13. Numerical streamlines (top) and isobars (bottom) for lid-driven cavity flow at $R e=10000$ : (a) Algorithm 1 and (b) Algorithm 2.

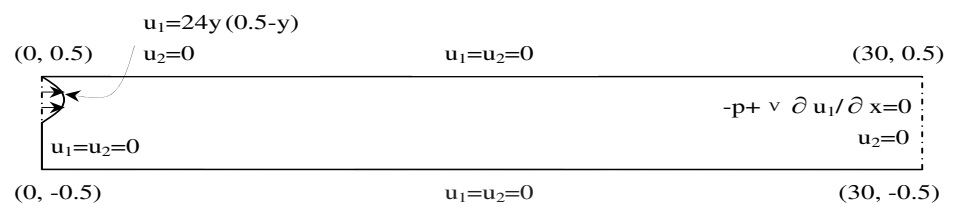

FiguRE 14. Schematic diagram of the backward-facing step flow.

The Taylor-Hood elements and the stopping criteria (5.2) and (5.3) are employed for Algorithms 1 and 2, respectively. Here the stability factor is set as $\alpha=0.004 h$, and the uniform mesh with size $h=1 / 20$ is adopted. In this test case, Algorithm 1 is only able to yield a numerical solution at Reynolds numbers up to 1000 due to its stricter stability condition, while Algorithm 2 works well at Reynolds numbers up to 1300. All the numerical results presented in this section were computed by Algorithm 2.

First, we compute the approximate solution at $R e=800$ and compare the results with those of Gartling [6]. Figure 15 plots the computed velocity, vorticity and pressure across the channel at $x=7$ and 15 compared with those of Gartling [6]. From Figure 15, we can see that when two-step corrections are performed, the computed vertical velocity at $x=7$ is not so accurate, while 


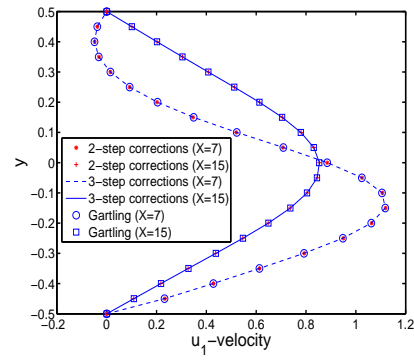

(a) $u_{1}$-velocity

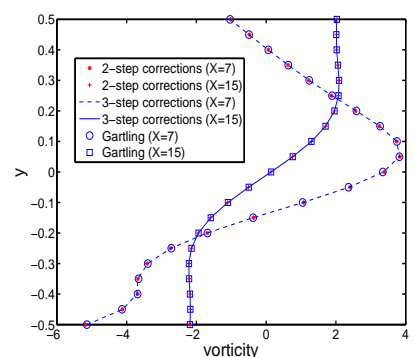

(c) vorticity

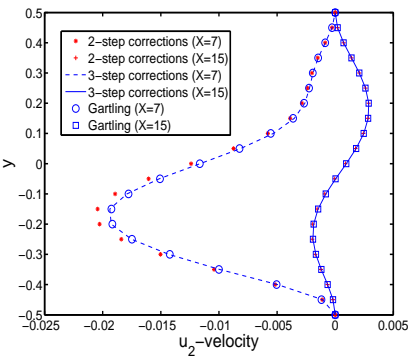

(b) $u_{2}$-velocity

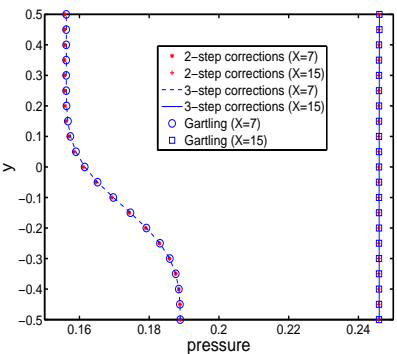

(b) pressure

FIGURE 15. Comparison of the computed velocity, vorticity and pressure at various downstream locations for the backward-facing step flow at $R e=800$.
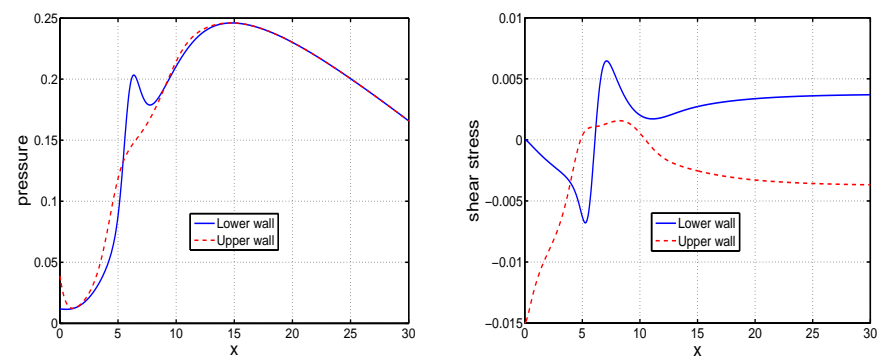

Figure 16. Pressure profiles (left) and shear stress profiles (right) along the upper and lower channel walls for the backward-facing step flow at $R e=800$.

three-step corrections yield a much better accurate solution which is in perfect agreement with those of [6]. This test case verifies the analysis in Remark 4.5. 


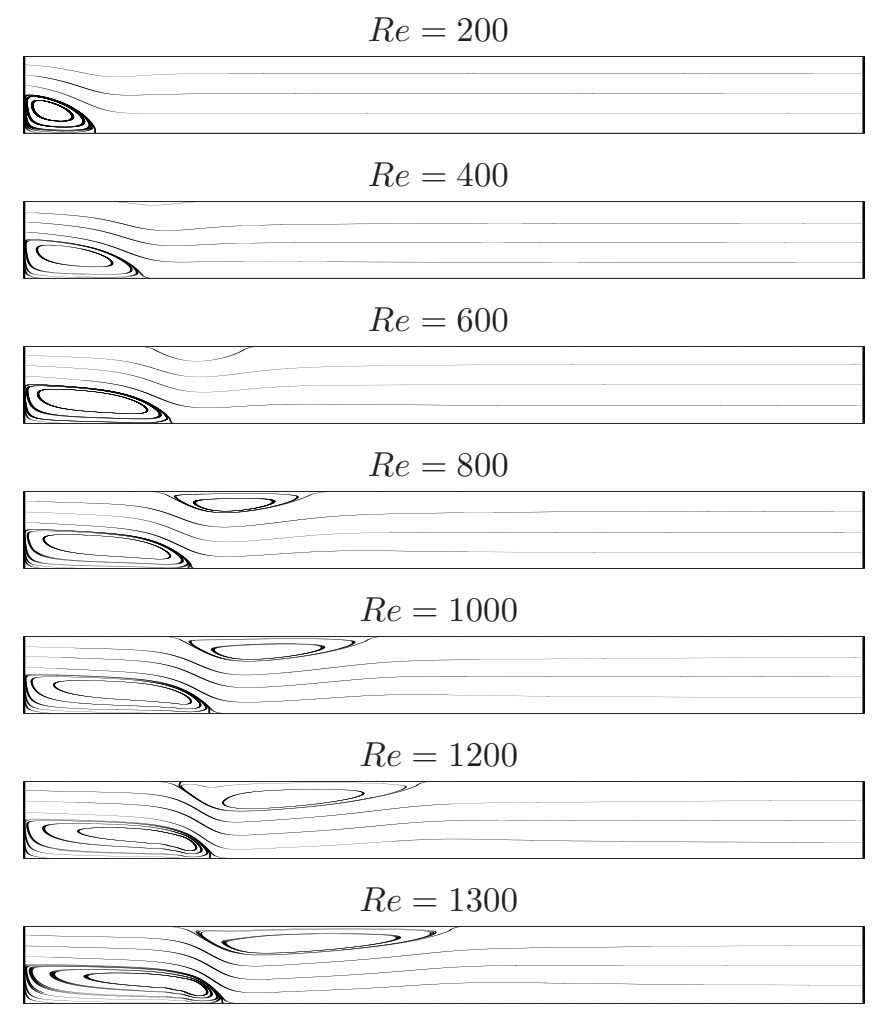

FiguRe 17. Numerical streamlines for the backward-facing step flow at various Reynolds numbers.

Figure 16 describes the computed pressure and shear stress along the upper and lower channel walls, which also coincide well with those of Gartling [6].

Figures 17 and 18 depict the computed streamlines and isobars at various Reynolds numbers, respectively, where the vertical $y$-scale is expanded to see the details. Figure 17 clearly shows that the length of the main recirculation region just after the step increases as the Reynolds number grows. At $R e=400$, a second recirculation eddy forms on the upper wall, which becomes larger as the Reynolds number further grows.

\section{Conclusions}

Based on finite element discreziation, two linearized defect-correction algorithms for the steady incompressible Navier-Stokes equations are introduced and analyzed in this work. The algorithms consist of an initial stabilization step where an artificial viscosity stabilized nonlinear Navier-Stokes problem is solved approximately by applying $m$ times of Newton or Picard iteration, and 


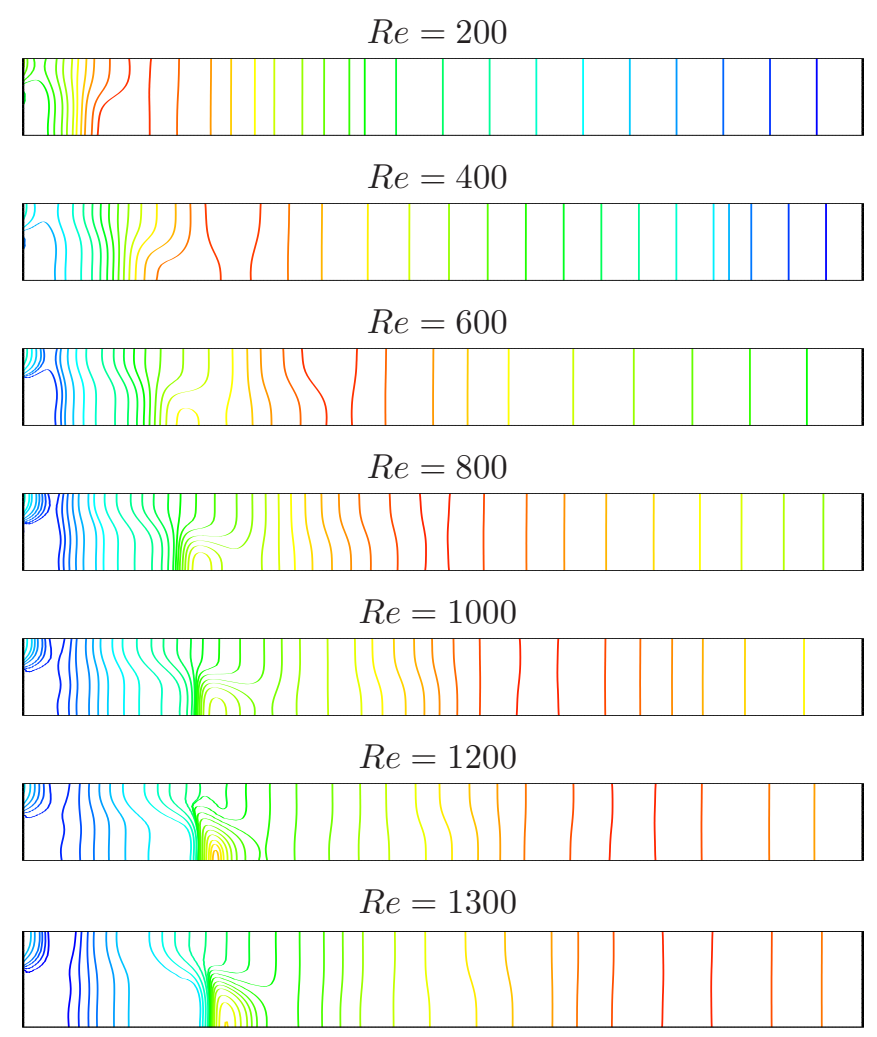

Figure 18. Numerical isobars for the backward-facing step flow at various Reynolds numbers.

several-step corrections where only a linear problem is solved at each step. Error estimates with respect to the mesh size $h$, the kinematic viscosity $\nu$, the stability factor $\alpha$ and the number of nonlinear iterations $m$ for the discrete velocity and pressure are derived for the one-step defect-correction algorithms. Stopping criteria for the nonlinear iterations and their influence on the accuracy of the approximate solutions are also investigated. Numerical simulations of a simple problem with known analytical solution, the lid-driven cavity flow, and the flow over a backward-facing step verify the theoretical results and demonstrate the effectiveness of the algorithms. Between the two linearized defect-correction algorithms, the Newton iterative algorithm is of second-order convergence rate with respect to the iterative steps, while the Picard iterative algorithm is more applicable in the simulations of high Reynolds number flows due to its weaker stability condition. 


\section{References}

[1] R. Adams, Sobolev Spaces, Academic Press Inc., New York, 1975.

[2] D. N. Arnold, F. Brezzi, and M. Fortin, A stable finite element for the Stokes equations, Calcolo 21 (1984), no. 4, 337-344.

[3] H. C. Elman, D. J. Silvester, and A. J. Wathen, Finite Elements and Fast Iterative Solvers: with Applications in Incompressible Fluid Dynamics, Oxford University Press, Oxford, 2005.

[4] E. Erturk, T. Corke, and C. Gokcol, Numerical solutions of 2-D steady incompressible driven cavity flow at high Reynolds numbers, Int. J. Numer. Methods Fluids 48 (2005), $747-774$.

[5] V. J. Ervin, W. Layton, and J. M. Maubach, Adaptive defect-correction methods for viscous incompressible flow problems, SIAM J. Numer. Anal. 37 (2000), no. 4, 11651185.

[6] D. K. Gartling, A test problem for outflow boundary conditions-flow over a backwardfacing step, Int. J. Numer. Methods Fluids 11 (1990), 953-967.

[7] U. Ghia, K. Ghia, and C. Shin, High-Re solutions for incompressible flow using the Navier-Stokes equations and a multigrid method, J. Comput. Phys. 48 (1982), 387-411.

[8] V. Girault and P. A. Raviart, Finite Element Approximation of the Navier-Stokes Equations, Springer-Verlag, Berlin, 1979.

[9] — Finite Element Methods for Navier-Stokes Equations: Theory and Algorithms, Springer-Verlag, Berlin, Heidelberg, 1986.

[10] R. Glowinski, Finite Element Methods for Incompressible Viscous Flow, in: P. G. Ciarlet and J. L. Lions (Ed.), Handbook of Numerical Analysis, Vol. IX, Numerical Methods for Fluids (Part 3), Elsevier Science Publisher, Amsterdam, 2003.

[11] Y. N. He, A fully discrete stabilized finite-element method for the time-dependent Navier-Stokes problem, IMA J. Numer. Anal. 23 (2003), no. 4, 665-691.

[12] Y. N. He and J. Li, Convergence of three iterative methods based on finite element discretization for the stationary Navier-Stokes equations, Comput. Meth. Appl. Mech. Engrg. 198 (2009), 1351-1359.

[13] Y. N. He and A. W. Wang, A simplified two-level method for the steady Navier-Stokes equations, Comput. Methods Appl. Mech. Engrg. 197 (2008), no. 17-18, 1568-1576.

[14] F. Hecht, O. Pironneau, and K. Ohtsuka, FreeFem++, Version 3.9-2, http://www. freefem.org. [2010-12-08]

[15] J. G. Heywood and R. Rannacher, Finite element approximation of the nonstationary Navier-Stokes problem I: Regularity of solutions and second-order error estimates for spatial discretization, SIAM J. Numer. Anal. 19 (1982), no. 2, 275-311.

[16] S. Kaya, W. Layton, and B. Riviere, Subgrid stabilized defect correction methods for the Navier-Stokes equations, SIAM J. Numer. Anal. 44 (2006), no. 4, 1639-1654.

[17] A. Labovschii, A defect correction method for the time-dependent Navier-Stokes equations, Numer. Methods Partial Differential Equations 25 (2009), no. 1, 1-25.

[18] W. Layton, Solution algorithm for incompressible viscous flows at high Reynolds number, Vestnik Mosk. Gos. Univ. Ser. 15 (1996), 25-35.

[19] W. Layton, H. Lee, and J. Peterson, A defect-correction method for the incompressible Navier-Stokes equations, Appl. Math. Comput. 129 (2002), no. 1, 1-19.

[20] H. K. Lee, Analysis of a defect correction method for viscoelastic fluid flow, Comput. Math. Appl. 48 (2004), no. 7-8, 1213-1229.

[21] Q. F. Liu and Y. R. Hou, A two-level defect-correction method for Navier-Stokes equations, Bull. Aust. Math. Soc. 81 (2010), no. 3, 442-454.

[22] Z. Y. Si, Y. N. He, and K. Wang, A defect-correction method for unsteady conduction convection problems I: spatial discretization, Sci. China Math. 54 (2011), no. 1, 185-204. 
[23] Z. Y. Si, Y. N. He, and T. Zhang, A defect-correction method for unsteady conduction convection problems II: Time discretization, J. Comput. Appl. Math. 236 (2012), no. 9, 2553-2573.

[24] C. Taylor and P. Hood, A numerical solution of the Navier-Stokes equations using the finite element technique, Comput. Fluids 1 (1973), no. 1, 73-100.

[25] R. Temam, Navier-Stokes Equations: Theory and Numerical Analysis, North-Holland Publishing Co., Amsterdam-New York-Oxford, 1977.

[26] K. Wang, A new defect correction method for the Navier-Stokes equations at high Reynolds numbers, Appl. Math. Comput. 216 (2010), no. 11, 3252-3264.

YUEQIANG SHANG

School of Mathematics and Statistics

SOUTHWEST UNIVERSITY

ChONGQING, 400715, P. R. China

E-mail address: yueqiangshang@gmail.com

DO WAN KIM

Department of Mathematics

INHA UNIVERSITY

INCHEON 402-751, KOREA

E-mail address: dokim@inha.ac.kr

TAE-Chang Jo

Department of Mathematics

INHA UNIVERSITY

INCHEON 402-751, KOREA

E-mail address: taechang@inha.ac.kr 